\title{
ISOTOPIC DEPENDENCE OF GCR FLUENCE BEHIND SHIELDING
}

\author{
Francis A. Cucinotta ${ }^{1, *}$, John W. Wilson ${ }^{2}$, Premkumar Saganti ${ }^{3}$, Xiaodong $\mathrm{Hu}^{1}$, \\ Myung-Hee Y. Kim ${ }^{1}$, Timothy Cleghorn ${ }^{1}$ \\ Cary Zeitlin ${ }^{4}$, Ram K. Tripathi ${ }^{2}$ \\ ${ }^{1}$ NASA, Lyndon B. Johnson Space Center, Houston TX, 77058 \\ ${ }^{2}$ NASA, Langley Research Center, Hampton VA, 23664 \\ ${ }^{3}$ Prairie View A\&M, Prairie View, TX, 94720 \\ ${ }^{4}$ Lawrence Berkeley National Laboratory Berkeley, CA 94720
}

* Corresponding author:

Dr. Francis A. Cucinotta, Chief Scientist

NASA Johnson Space Center

Houston TX, 77058

Francis.A.Cucinotta@nasa.gov

Phone: 281-483-0968

Fax: 291-483-3058 


\begin{abstract}
:
In this paper we consider the effects of the isotopic composition of the primary galactic cosmic rays (GCR), nuclear fragmentation cross-sections, and isotopic-grid on the solution to transport models used for shielding studies. Satellite measurements are used to describe the isotopic composition of the GCR. For the nuclear interaction data-base and transport solution, we use the quantum multiple-scattering theory of nuclear fragmentation (QMSFRG) and high-charge and energy (HZETRN) transport code, respectively. The QMSFRG model is shown to accurately describe existing fragmentation data including proper description of the odd-even effects as function of the iso-spin dependence on the projectile nucleus. The principle finding of this study is that large errors $( \pm 100 \%)$ will occur in the mass-fluence spectra when comparing transport models that use a complete isotopic-grid ( $\sim 170$ ions) to ones that use a reduced isotopicgrid, for example the 59 ion-grid used in the HZETRN code in the past, however less significant errors $(< \pm 20 \%)$ occur in the elemental-fluence spectra. Because a complete isotopic-grid is readily handled on small computer workstations and is needed for several applications studying GCR propagation and scattering, it is recommended that they be used for future GCR studies.
\end{abstract}

Keywords: Space radiation shielding, nuclear fragmentation, and galactic cosmic rays. 


\section{INTRODUCTION}

An important goal for NASA's Space Radiation Health Program is to develop a predictive capability to predict the GCR fluence spectra to within a $\pm 25 \%$ accuracy (Annon. 1998). NASA has developed the HZETRN (high-charge and energy transport) code (Wilson, 1977; Wilson and Badavi, 1986; Wilson et al., 1991) as a science application and engineering design tool (Wilson et al., 1993) to be used in space radiation shielding studies. HZETRN has been validated in its ability to predict total dose and dose equivalent behind several materials in space to within $\pm 20 \%$ on multiple space missions in Earth orbit (Cucinotta et al., 2000a, Badhwar and Cucinotta, 2000, Badhwar et al., 2001). However, interest in fluence-based approaches to risk assessment (Cucinotta et al., 1995a, 1996a) suggests that more stringent tests of transport code accuracy be made, and the quantities dose and dose equivalent are deemed as necessary, but not sufficient tests of their accuracy. In this regard, we note that dose and dose equivalent are integral quantities that receive contributions from many GCR charge groups. There currently exist large uncertainties in biological response models for GCR (Cucinotta, et al., 2001) and methodologies to estimate health risks such that dose and dose equivalent may be insufficient as tests of transport code accuracy. The use of ion fluence as a basis for tests for accuracy provides for sufficient generality to ensure accuracy in GCR transport models including under the circumstances of revision of radiation quality factors or integration of alternative risk assessment approaches in the future.

In the description of the transport of the galactic cosmic rays (GCR) in shielding materials or tissue, a common approximation is to consider only the elemental composition of the primary GCR and a reduced isotopic-grid for the secondary nuclei produced in nuclear fragmentation. In this paper we analyze the role of the isotopic dependence of the GCR primary composition and nuclear fragmentation in predicting the fluence of the GCR behind arbitrary shielding configurations. Our study is an important milestone in achieving NASA's goal of accurate GCR transport codes, since for the firsttime a complete isotopic-grid has been achieved in a GCR transport model and we document the error inherent in former approaches. Also, for applications that will consider radioactive isotopes produced in the atmosphere or shielding, our study provides 
a useful tool to perform such analyses. Other applications where non-stable nuclei are considered are studies of the origin or the GCR where so-called cosmic-ray "clocks" consider the primary or secondary GCR with life-times on the order of confinement time in the galaxy ( 1 M-yrs) (Yanasak et al., 1999). Several GCR "clock" nuclei including ${ }^{10} \mathrm{Be}$ and ${ }^{26} \mathrm{Al}$ were not considered in the grid used in HZETRN in the past. Finally new data on the GCR near Mars are being collected by the MARIE experiment on the Odyssey spacecraft (Zeitlin et al., 2004), and our study provides an opportunity to begin new investigations on the accuracy of computational models used to describe the GCR.

Historically the HZETRN code grew from a 29-ion isotopic grid used in the 1980's and early 1990's (Wilson, et al., 1991) to an extension to a 32-ion isotopic grid made in 1993 in order to include all light ions (Cucinotta, 1993). Because of the limitations of random access memory present on the computer workstations of the early 1990's, sensitivity studies were made for mono-energetic ion beams to study the minimum number of isotopes for convergence resulting in the use of 59-isotopic grid (Kim, et al., 1994), and all GCR studies since 1994 have used the 59-isotopic grid (Shinn, et al., 1994). However, there are several reasons to re-consider the use of the full isotopic-grid for GCR transport problems. First, the isotopic dependence of the primary GCR has not been considered in past shielding studies and may lead to errors in the description of both primary ion attenuation and secondary particle production including the role of high-energy neutron production from the many neutron rich species that occur. Secondly, the studies of Kim et al. (1994) used the NUCFRG2 model of fragmentation (Wilson et al., 1994), which does not provide a correct description of the even-odd effect observed in fragment production or of the projectile iso-spin dependence observed experimentally (Knott et al., 1996, 1997; Zeitlin et al., 2001). Thirdly, the sensitivity studies made by Kim et al. (1994) used a "calibration" of the isotopic-grid to ${ }^{56} \mathrm{Fe}$ beams; however a larger isotopic grid occurs when all GCR projectile nuclei are considered. Fourth, the error in the range-energy and stopping powers that results from the use of a reduced isotopic grid, although expected to be small for large mass number, $\mathrm{A}>>1$, is an unnecessary one for transport calculations. Finally, the improved computational speed and memory available on current small 
computer workstations, makes the inclusion of a complete isotopic-grid in the HZETRN code to be readily implemented at this time.

In this paper the implementation of the HZETRN code to include the full isotopic dependence of the primary GCR is described. The physics of isotopic effects in GCR transport are described and the fragmentation parameters are a key component of this description. The quantum multiple scattering theory of nuclear fragmentation (QMSFRG) is used as the generator for fragmentation cross-sections used in our study. An empirical model of the isotopic composition of the primary GCR including its solar modulation is also described. For GCR problems an isotopic grid of 170 ions is identified and comparisons made to previous HZETRN results using the reduced-grid of 59 isotopes are made. The present code includes all of the abundant nuclei in the GCR environment with fluxes greater than about $10^{2} / \mathrm{cm}^{2} / \mathrm{yr}$ and nuclei produced in fragmentation events with production cross-sections greater than about $1 \mathrm{mb}$. Several nuclei with smaller primary abundances or production cross-sections, which are of interest for scientific reasons are also included in the expanded HZETRN model described herein. The resultant code includes many neutron rich nuclei that have been ignored in the past with iso-spin components ranging from $T_{2}=+3 / 2$ to $T_{2}=-3$.

\section{ISOTOPIC COMPOSITION OF THE GCR}

NASA currently uses the GCR model of Badhwar and O'Neill (1992) to describe the elemental composition and energy spectra of the GCR including their modulation by the sun's magnetic field. In this GCR representation, only the most abundant GCR nuclei is considered for each element and other isotopes of identical charge are counted as the abundant isotope. However, theoretical models and satellite measurements of the GCR have long considered the isotopic composition of the GCR and their modification through transport in interstellar space including estimating the primary nuclear composition at stellar sources (Parker, 1965, Webber et al., 1990a, Fields et al., 1994). The approach used here is to estimate an energy-independent isotopic fraction, $f_{j}$ from satellite measurements, which are constrained to obey the sum-rule 
(1) $\phi(Z, E)=\sum_{A_{j}} f_{j}\left(A_{j}, Z\right) \phi\left(A_{j}, Z, E\right)$

where the left-hand side of eq. (1) is the elemental spectra from the Badhwar and O'Neill model and $\sum_{j} f_{j}=1$. Equation (1) is used herein as an initial estimate of the influence of the primary isotopic composition on GCR shielding calculations. Experimental studies have included measurements on the Pioneer, Voyager, and Ulysses spacecraft. A survey of such data (Hesse et al., 1991; Lukasiak, et al., 1993, 1995; Webber et al., 1985, 1990; Wiedenback et al, 1981, 1985) was made with the results shown in Table-1.

The GCR path-length distribution represents the mean amount of inter-stellar and interplanetary material intersected by cosmic rays prior to their arrival in the near-Earth environment. This distribution is currently estimated to vary between 3 and $20 \mathrm{~g} / \mathrm{cm}^{2}$ with the inter-planetary gas approximately $90 \% \mathrm{H}$ and $10 \% \mathrm{He}$. Variability in estimates between various studies arise because of differences in model nuclear fragmentation parameters, cosmological interaction terms, and factors related to the near-Earth or deep space measurements including energy and mass resolution of the detectors as well as position in the solar cycle. In most current models, the GCR path-length distribution is estimated to be velocity and rigidity dependent (i.e., energy dependent). Of note is that based on the solar modulation theory of Parker (1965), the inter-planetary portion of this distribution would be modulated over the solar cycle suggesting the form

(2) $\lambda(\Phi, R)=\lambda_{\text {Inter-stellar }}+\lambda_{\text {Inter-planetary }}(\Phi, R)$

where $\Phi$ is the solar modulation parameter and $R$ (in A.U.) is the radial distance from the sun. It then follows that the amount of fragmentation in the inter-planetary media and the isotopic abundances are dependent on the modulation parameter and radial distance. Based on the results of Lukasiak et al. (1993) the following formula is used to describe the dependence of the isotopic ratio (near-Earth) on the solar cycle including a dependence on the modulation parameter $\Phi(\mathrm{MV})$ 
(3) $f(A, Z)=f_{\text {source }}(A, Z)+[\exp (\gamma \sqrt{\Phi})-1]$

where $\gamma$ is found by interpolating from the source and near solar maximum values. The second term in Eq.(3) can be positive or negative as dependent on the conditions for a net gain or reduction in the cosmic nuclei during transit from the galactic sources (Fields et al., 1994) to the inner heliosphere. In Table-1 we have used data on isotopic fractions near solar maximum where the isotopic fraction for nuclei produced within the heliosphere are expected to be at a maximum (Hesse et al., 1991; Lukasiak et al., 1993; Webber et al., 1985, 1990; Wiedenback et al, 1981, 1985). For several elements listed in Table-1, information on solar modulation was not available and isotopic fractions for near-Earth and GCR source were set identical using the near-Earth estimate.

We represent the primary (near-Earth) ${ }^{2} \mathrm{H}$ and ${ }^{3} \mathrm{He}$ spectra as

$$
\begin{aligned}
\phi_{3 \mathrm{He}}(E) & =\left\{0.0764+0.097 \exp \left[-0.5(\ln (E / 1660) / 1.306)^{2}\right]\right\} \phi_{4 \mathrm{He}}(E) \\
\phi_{2_{H}}(E) & =0.2 \phi_{{ }^{H} \mathrm{He}}(E)
\end{aligned}
$$

where $\phi_{3 \mathrm{He}}(E)$ and $\phi_{2 \mathrm{H}}(E)$ are subtracted from the Badhwar and O'Neill model (1992) for $\phi_{4 \mathrm{He}}(E)$ and $\phi_{1 \mathrm{H}}(E)$, respectively. Figure-1 shows the assumed dependence of the isotope abundance scaling as a function on the solar modulation parameter described by equation (3) for ${ }^{13} \mathrm{C}$ and ${ }^{15} \mathrm{~N}$. Similar results are found for other primary isotopes listed in Table-2. Examples of the GCR energy spectra for hydrogen and helium are shown in Figure-2a and for the isotopes of $\mathrm{Ne}, \mathrm{Si}$, and $\mathrm{Fe}$ at solar minimum in Figure-2b.

\section{ISOTOPIC EFFECTS IN GCR TRANSPORT}

The reduction of the full 3-dimensional Boltzmann transport equation within the straightahead and continuous slowing down approximations and assuming velocity conservative fragmentation events accurately describes the transport of GCR heavy ions (Wilson et al., 
$1986,1991,1995)$. In this model the heavy ion flux, $\phi_{j}(E, x)$ of an ion $j$ with mass number $A_{j}$, charge number $Z_{j}$, energy $E$ (in units of $M e V / u$ ) at shielding depth $x$ (in units of $\mathrm{g} / \mathrm{cm}^{2}$ ) is determined by the partial differential equation (Wilson et al., 1991)

$$
\left[\frac{\partial}{\partial x}-\frac{1}{S_{j}(E)} \frac{\partial}{\partial E}+\sigma_{j}(E)\right] \phi_{j}(x, E)=\sum_{k} \sigma_{j, k}(E) \phi_{k}(E, x)
$$

where $\sigma_{j}(E)$ is the energy-dependent absorption cross-section $\left(\mathrm{cm}^{-1}\right)$ and $\sigma_{j k}(\mathrm{E})$ is the fragmentation cross-section for producing an ion $j$ from $k$. The solution to eq. (5) in the HZETRN code is found using the methods of characteristics where the coordinate transformation

(6a) $\eta_{j}=x-R_{j}(E) ; \quad \xi_{j}=x+R_{j}(E)$

and the scaled flux

$$
\chi_{j}\left(\eta_{j}, \xi_{j}\right)=S_{j}(E) \phi_{j}(x, E)
$$

are introduced leading to the transport equation

$$
\left[2 \frac{\partial}{\partial \eta_{j}}+\sigma_{j}\right] \chi\left(\eta_{j}, \xi_{j}\right)=\sum_{k} \sigma_{j, k} \frac{v_{j}}{v_{k}} \chi\left(\eta_{k}, \xi_{k}\right)
$$

where $S_{j}(E)$ is the stopping power of ion $j, v_{j}=Z_{j}^{2} / A_{j}, \sigma_{j}$ the total absorption crosssection, and $\sigma_{j k}$ the fragmentation cross-section to produce isotope $j$ from projectile isotope $k$. The numerical solution to Eq.(7) uses a marching procedure (Wilson et al. (1990)). The solution of the transport equation for light ions and neutrons is distinct from Eq.(7) because of the broad re-distribution of energy in collision events and is described elsewhere (Wilson et al., 1991, Cucinotta et al., 1995b, and Clowdsley et al., 2000).

Because Eq.(7) is a coupled integro-differential equation for the many GCR primary and secondary nuclei, required computer memory allocations increase rapidly as the number of ion species is increased and was an important consideration in the early 1990's. 
However, such practical limitations no longer exist, even on small computer workstations with sufficient RAM (e.g. Pentium III or higher with 0.5 Giga-bytes or more), and an unrestricted isotopic-grid can presently be implemented with no memory or storage problems. When using a reduced isotopic grid, fragments not contained in the grid are assigned to a nearby mass of identical charge number. This introduces two types of errors: First the range-energy and stopping powers are altered from their true values. This error is expected to be small at high-energies for $A \gg>1$ since here ion ranges are well described by $A_{j} / Z_{j}^{2}$ scaling factors. The second type of error occurs in the absorption and fragmentation cross-sections. Here because of the re-assignment of the mass number when using a reduced grid, an error is introduced by the change in neutron number from its true values. The fluence spectra of high-energy neutrons produced in fragmentation events is also modified by forcing the physics onto a reduced isotopic-grid. This latter error is expected to be small for heavy target nuclei $(A>16)$ because neutron production is dominated by light-particle ( $n, p, d, t, h$, and $\alpha$ ) interactions on target nuclei, but may be non-negligible for light target atoms $(A \leq 16)$ (Cucinotta et al. 1998a). Since materials with high-hydrogen content are known to be the optimal shielding materials, the changes in the neutron fluence due to the use of a full isotopic-grid should be considered.

\section{QUANTUM FRAGMENTATION MODEL}

We next discuss the quantum multiple scattering description of heavy ion fragmentation (QMSFRG), which has been quite successful in describing the physics of the abrasion ablation model of fragmentation and experimental data (Cucinotta, et al. 1992, 1994a, 1997, 1998b). For inclusive reactions where a single fragment originating in the projectile is measured, closure is performed on the final target state with a momentum vector denoted $\mathbf{p}_{X}$ used to represent these states. The total momentum transfer is $\mathbf{q}=\mathbf{p}_{T}-\mathbf{p}_{X}$ where $\boldsymbol{p}_{T}$ is the initial target momentum. The pre-fragment, $F^{*}$ excitation spectrum following nucleon or alpha particle abrasion can be represented in terms of an impact parameter dependent convolution of the pre-fragment excitation response for a transition of the pre-fragment core from state $n$ to $n$ ' and the project fireball response (Cucinotta et al., 1994a, 1998b) 


$$
\frac{d \sigma}{d E_{F^{*}}}=<T\left|\int d^{2} q d^{2} b d^{2} b^{\prime} \quad e^{i \mathbf{q}\left(\mathbf{b}-\mathbf{b}^{\prime}\right)} P_{n, n^{\prime}}\left(b, b^{\prime}\right) \Lambda_{n, n^{\prime}}\left(q, b, b, E_{F^{*}}\right)\right| T>
$$

where $b\left(b^{\prime}\right)$ is the impact parameter, and $q$ the momentum transfer. The abrasion response is defined as the interaction of the projectile fireball with the target after performing closure over the final fireball states

$$
\Lambda_{n, n^{\prime}}\left(b, b^{\prime}, E_{i f}\right)=\int \frac{d \mathbf{k}_{R}}{(2 \pi)^{3}} \quad<R^{\prime}\left|Q_{R T}{ }^{+}\left(b^{\prime}\right)\right| R><R\left|Q_{R T}(b)\right| R^{\prime}>\delta\left(E_{i}-E_{f}\right)
$$

where the $Q_{R T}$ represent the fireball-target profile operator, and $k_{R}$ the projectile fireball momentum vector. The abrasion-response represents a complicated many-body operator that is solved by approximation using closure over the target and fireball states for evaluating the pre-fragment distribution. The one-particle abrasion response has been evaluated using the shell model response functions (Cucinotta and Dubey, 1993). The pre-fragment excitation is described in terms of the transition matrix

$$
P_{n, n^{\prime}}\left(b, b^{\prime}, E_{F^{*}}\right)=<F_{n}^{*}\left|Q_{F^{*} T}^{+}\left(b^{\prime}\right)\right| F_{n^{\prime}}^{*}><F_{n^{\prime}}^{*}\left|Q_{F^{*} T}(b)\right| F_{n}^{*}>
$$

where matrix elements for the pre-fragment excitation are evaluated over the many-body profile operators, $Q_{F T}$. In the model a convolution approach is used to derive the mutliknockout spectrum from the single-fragmentation term (Cucinotta and Dubey, 1994a). The resulting excitation spectrum is broad with a shape similar to a log-normal distribution with mean energies from 20 to $30 \mathrm{MeV}$ for one-nucleon removal (Cucinotta and Dubey, 1993; 1994a).

The de-excitation of the pre-fragments in nuclear ablation is described in a stochastic process using a Master equation for nuclear de-excitation by particle emission (Cucinotta and Wilson, 1996b). If $f^{b}(E, t)$ is the probability of finding the nuclei $b$ at time $t$ with excitation energy $E_{b}$ and $P_{k}^{b}(E)$ be the probability that the nuclei, $b$ will emit ion $k$ with energy $E$, then the Master equation is 
(11) $\frac{d f^{b}\left(E_{b}^{*}, t\right)}{d t}=\sum_{j} \int d E f^{a}\left(E_{a}^{*}, t\right) P_{j}^{a}(E)-\sum_{k} \int d E f^{b}\left(E_{b}^{*}, t\right) P_{k}^{b}(E)$

In Eq.(11) the first-term on the right corresponds to gains by decays $a->b+j$ and the second term from losses due to decays $b->c+k$ where the $j$ (or $k$ ) are light-particle emissions ( $n, p, d, t, h$, or $\alpha$ ). Equation (11) is solved by iteration up to excitation energies of $150 \mathrm{MeV}$ and by approximation for higher values (Cucinotta et al. 1996b, 1997a). Important features of this solution is the correct description of the nuclear leveldensity including nuclear-shell effects at low-excitation energies, and the use of measured values for the nuclear masses. The fragmentation cross-section is then evaluated from as

$$
\sigma_{F}\left(A_{F}, Z_{F}\right)=\sum_{A_{F}^{*}, Z_{F}^{*}} \int_{F^{*}} \frac{d \sigma_{F^{*}}}{d E_{F^{*}}} f\left(A_{F^{*}}, Z_{F^{*}} \longrightarrow A_{F}, Z_{F}\right)
$$

where $f\left(A_{F^{*}}, Z_{F^{*}} \longrightarrow A_{F}, Z_{F}\right)$ is the solution to Eq.(11).

The iso-spin dependence in fragmentation cross-sections enters in several ways. First, in the many-body profile functions, the energy-dependent two-body nucleon interaction parameters are summed over the possible projectile and target nucleon scattering combinations. Second, the nuclear wave-functions are dependent on shell structure. The largest contribution to iso-spin effects occur in the nuclear ablation process (deexcitation) where the pre-fragments formed and their level spectra are greatly influenced by the projectile or pre-fragment iso-spin. The model uses Coulomb trajectories (Cucinotta et al., 1997a) and in-medium nucleon-nucleon interaction (Tripathi et al., $2000)$, which are important at lower energies $(<200 \mathrm{MeV} / \mathrm{u})$.

\section{RESULTS}

We first illustrate the accuracy of the QMSFRG model and the effects of iso-spin on fragmentation cross-sections. Figures 3 and $\mathbf{4}$ show comparisons of the model to experimental data for the elemental distributions of fragments for several nuclei of 
similar mass number where nearby isotopes are chosen for comparison. The data of Webber et al. (1998) used fragmentation of a primary beam and steering magnets to form several less abundant nuclei, and we note the beams are only about $90 \%$ pure for ${ }^{29} \mathrm{Si}$, ${ }^{46} \mathrm{Sc}$, and ${ }^{46} \mathrm{Ti}$ results shown. In all comparisons the one-nucleon removal cross-sections by electro-magnetic dissociation are included (Norbury et al., 1988). The iso-spin, $T_{z}=0$ nuclei display large odd-even effects, which are reduced for the $T_{z} \neq 0$ nuclei. The oddeven effects are present for all target nuclei, however smaller for hydrogen targets due to the reduced abrasion probability for large mass removal on hydrogen. One of errors that results from transporting ions using a reduced mass-grid is seen by comparing fragmentation cross-sections for nearby projectiles where large differences in many of the production cross-sections occur for neighboring projectile nuclei. The model accurately reproduces the effects observed in the experiments. Figures-5 show comparisons of QMSFRG predictions to experimental data for the isotopic distribution of fragments from ${ }^{40} \mathrm{Ar}$ and ${ }^{56} \mathrm{Fe}$, and Figures 6-8 correlations between the model and experimental data for ${ }^{20} \mathrm{Ne},{ }^{32} \mathrm{~S},{ }^{36} \mathrm{Ar}$, and ${ }^{40} \mathrm{Ar}$ and ${ }^{56} \mathrm{Fe}$ elemental production cross sections. The results show good agreement between theory and experiments with the QMSFRG model within 25\% percent of the experimental values for over $90 \%$ of the data in the literature.

For our extension of the HZETRN code, Table-2 shows the isotopic table of 170 nuclei developed as a complete list of GCR primary and secondary nuclei to be used in GCR transport problems. We have also listed in the Table-2 the iso-spin, and half-life for the unstable nuclei along with the decay mode. This table of nuclei includes all nuclei of significant abundance with iso-spin $+3 / 2$ to -3 that appear in GCR transport problems. By contrast early versions of the HZETRN code used a much smaller ion table. The expanded grid used here will allow for improved description of the physics, and to discuss many applications where unstable nuclei are central to understanding. Also listed in the first column of Table-2 is the index scheme that is used in the HZETRN code, which is used to label the shielding-depth and energy dependent fluence matrix. The index for the nuclei are ordered by increasing mass number, $A$ followed by charge number, $Z$ for a given $A$. 
The GCR nuclei are completely stripped and therefore the decay mode and half-life for unstable nuclei could be differential from those observed in laboratories on Earth. GarciaMunoz et al. (1987) has noted the following differences: 1) electron capture branches, which are inactive for GCR nuclei relative to $\beta$-decay, 2) for non-stripped nuclei there will be two S-shell electrons that participate in electron capture that will not be available in the decay of GCR nuclei, and 3) changes in screening effects. They have made estimates of the elongation of the half-time for fully stripped nuclei due to these processes, which indicate an approximate doubling of the decay time observed for laboratory nuclei that normally decay by electron capture. Since these decay times are much longer than the transit time of nuclei in shielding they are not considered here. However, it will useful in the future to further consider these processes when studying the effects of stopping GCR nuclei on planetary atmospheres or surfaces and in tissues.

Figure-9 shows results from the HZETRN code at solar minimum ( $\Phi=428 \mathrm{MV})$ behind 5 $\mathrm{g} / \mathrm{cm}^{2}$ of aluminum shielding. Comparison of the mass-flux spectra for a 59-isotope grid and the 170-isotope grid are shown. The 170-isotope grid was developed by considering the fragmentation cross-sections for a large number of GCR primary nuclei and dominant fragments in several materials. In Figure-9 we have scaled the flux by the square of the ion charge as a measure of the ionization power of each mass group. Large differences are seen for many nuclei. Figure-10a shows the percent error in the mass-fluence spectra resulting from the use of the reduced grid for depths of 5 and $20 \mathrm{~g} / \mathrm{cm}^{2}$ of aluminum. Errors greater than $100 \%$ are seen for many nuclei, however in most cases such large errors only occur for the less abundant nuclei. The probability of biological effects are expected to increase in a manner proportional to $Z^{2}$ for a given energy, and the elementalflux distribution may be a sufficient test of transport models for supporting exploration studies. In Figure-10b we show a similar comparison to that of Figure-10b, however here for the elemental-fluence spectra. The errors are indeed less substantial than those of the mass-fluence spectra, yet are larger than $10 \%$ in several cases. Similar comparisons near solar maximum conditions are shown in Figures-11. In Table-3 results for the elemental and neutron excess dependence of the point dose equivalent behind aluminum shielding are shown. The $Y<-1$ nuclei are not significant, while all other cases make 
important contributions. Table-4 shows the fluence at several depths for several of the cosmic-ray clock nuclei and other less abundant nuclei of interest for scientific studies.

\section{DISCUSSION}

It has been recognized for many years that for the description of GCR transport in shielding, theoretical models and experimental data describing the nuclear interactions and propagation of protons, heavy ions and their secondaries, leading to accurate and computational efficient transport codes are needed. In the last 25 years such descriptions have improved dramatically with major milestones that include the development of an accurate free space GCR model (Badhwar and O'Neill, 1992), the HZETRN code (Wilson, 1977; Wilson, et al., 1990), the measurement of a significant number of fragmentation cross-sections (Brechtmann and Heinrech, 1988; Webber et al., 1990b Knott et al. 1996, 1997; and Zeitlin et al., 1997, 2001, 2002), and the development of an accurate nuclear fragmentation model (Cucinotta et al., 1998a). Laboratory (Shimmerling et al, 1989), and spaceflight (Badhwar and Cucinotta, 2000) validation data have also become available during this time period. The combination of the GCR model of Badhwar and O'Neill, QMSFRG cross section data base, and HZETRN transport code have been shown to agree with flight measurements of GCR dose and dose equivalent within $\pm 15 \%$ on several space vehicles. However, further spectral data sets, both in space and at heavy ion accelerators, are needed to fully validate these codes.

The implementation of heavy ion transport models has progressed from models that did not satisfy unitarity (Letaw et al., 1983), to the current fully energy-dependent models with accurate absorption cross-sections (Shinn et al., 1994; Wilson et al., 1993; Cucinotta, 1993). Future work may still be required for light-particle transport ( $n, p, d, t$, $h, \alpha$, and mesons and their decays), including establishing production cross-section models and data, and understanding the role of angular deflections, which are more important for neutrons than for heavy ion transport. However, the heavy ion problem is in much better shape with many of the remaining task ones of implementation. One exception may be improvements in fragmentation cross-sections and laboratory validation for the $Z=1$ to 5 nuclei produced from the heavier projectile nuclei $(Z>10)$. 
The present paper addressed two implementation tasks; the use of a free-space GCR model, which includes the isotopic composition of the primaries, and the extension of the HZETRN code to a complete isotopic-grid. The problem of the isotopic distribution of the primary GCR and their modulation during the solar cycle has been treated in a parametric way in this paper. In this approach, we maintain the accuracy of the GCR modulation model for the GCR elemental spectra from Badhwar and O'Neill (1992), however re-distribute the fluence of each element amongst its isotopes using estimates from satellite data. The coupling of the HZETRN code to the Leaky Box Model is a possible approach to a more exact treatment of this problem including the description of the energy dependence of the isotopic fractions. However, for shielding applications these effects are expected to be small based on the current study. The use of the complete isotopic grid of nuclei allows HZETRN applications on the study of the so-called cosmic ray "clocks" with lifetimes similar to the time spent by GCR nuclei in the galaxy $(\sim 1 \mathrm{M}$ yr) as well as studies of signature nuclei from the decay of GCR nuclei in the Mars atmosphere or planetary surfaces. Because the use of a reduced-grid leads to error and there are no practical limitations in using a complete isotopic-grid at this time, we recommend it be used when initiatives to design space exploration vehicles begin. Future tasks that remain are to implement physical models of the GCR isotopic environment and to continue to refine the QMSFRG model including comparisons to new fragmentation data as they become available. For the many deformed and highly deformed projectile nuclei considered herein, methods to consider this deformation in the abrasion process are needed. Other considerations for future work is to study the isotopic effects for other materials and to consider the decay of radioactive isotopes produced as GCR secondaries in the Mars atmosphere, shielding materials or tissue.

\section{REFERENCES}

Anonymous, 1998. Strategic Space Radiation Health Program Plan, NASA Office of Life Sciences and Microgravity Applications, Washington D.C.

Badhwar, G.D., O’Neill, P.M., 1992. An Improved Model of GCR for Space Exploration. Missions. Nucl. Tracks Radiat. Meas. 20, 403-410. 
Badhwar, G.D., Cucinotta, F.A., 2000. A Comparison on Depth Dependence of Dose and Linear Energy Transfer Spectra in Aluminum and Polyethylene. Radiat. Res. 153, 1-8.

Brechtmann, C., Heinrich, W., 1988. Fragmentation Cross-sections of ${ }^{32} \mathrm{~S}$ at $0.7,1.2$, and $200 \mathrm{GeV} /$ nucleon. Z.Phys. A331, 463-472.

Clowdsley, M., Wilson, J.W., Kim, M.H.Y., Singleterry, R.C., Tripathi, R.K., Heinbockel, J.H., Badavi, F.F., Shinn, J.L., 2001. Neutron Environments on the Martian Surface. Phys. Medica XVII 94-96.

Cucinotta, F.A., Townsend, L.W., Wilson, J.W., 1992. Multiple Scattering Effects in Quasi-Elastic $\alpha-{ }^{4}$ He Scattering. Phys. Rev. C 46, 1451-1456.

Cucinotta, F.A., 1993a. Calculations of Cosmic-Ray Helium Transport in Shielding Materials. NASA TP-3354.

Cucinotta, F.A., Dubey, R.R., 1993b. Final State Interactions and Inclusive Nuclear Collisions. NASA TP-3533.

Cucinotta, F.A., Dubey, R.R., 1994a. Alpha Cluster Description of Excitation Energies in ${ }^{12} \mathrm{C}\left({ }^{12} \mathrm{C}, 3 \alpha\right) \mathrm{X}$ at $2.1 \mathrm{GeV}$. Phys. Rev. C50, 979-984.

Cucinotta, F.A., 1994b. Forward Production of Protons in Relativistic ${ }^{12} \mathrm{C}$-nucleus Collisions. J. Phys. G: Nucl. Part. Phys. 20, 1803-1815.

Cucinotta, F.A., Wilson, J.W., 1995a. Initiation-Promotion Model of Tumor Prevalence in Mice from Space Radiation Exposure. Radiat. Environ. Biophys. 34, 145-149.

Cucinotta, F.A., Townsend, L.W., Wilson, J.W., Shinn, J.L., Badhwar, G.D., Dubey, R. R., 1995b. Light Ion Components of the Galactic Cosmic Rays: Nuclear Interactions and Transport. Adv. in Space Res. 17, 77-86.

Cucinotta, F.A., Wilson, J.W., Shavers, M.R., Katz, R., 1996a. The Effects of Track Structure and Cell Inactivation on the Calculation of Heavy Ion Mutation Rates in Mammalian Cells. Inter. J. Radiat. Biol. 69, 593-600.

Cucinotta, F.A., Wilson, J.W., 1996b. Study of Analytical Statistical Model for Decay of Light and Medium Mass Nuclei in Nuclear Fragmentation. NASA TP-3594.

Cucinotta, F.A., et al., 1997a. Computational Procedures and Data-Base Development. In: NASA Workshop on Shielding Strategies for Human Space Exploration. Eds. Wilson J.W., Miller J., Konradi A., and Cucinotta F.A.. NASA CP-3360.

Cucinotta, F.A., Wilson, J.W., Townsend, L.W., 1997b. Abrasion-Ablation Model for Neutron Production in Heavy Ion Collisions. Nucl. Phys. A. 619, 202-212. 
Cucinotta, F.A., Wilson, J.W., Tripathi, R.K., Townsend, L.W., 1998. Microscopic Fragmentation Model for Galactic Cosmic Ray Studies. Adv. in Space Res. 22, 533-537.

Cucinotta, F.A., Wilson, J.W., Williams, J.R., Dicello, J.F., 2000. Analysis of Mir-18 Results For Physical and Biological Dosimetry: Radiation Shielding Effectiveness in LEO. Radiat. Meas. 132, 181-191.

Cucinotta, F.A., Schimmerling, W., Wilson, J.W., Peterson, L.E., Badhwar, G.D., Saganti, P., Dicello, F.J., 2001. Space Radiation Cancer Risks And Uncertainties For Mars Missions. Radiat. Res. 156, 682-688 (2001).

Cummings, J.R., Binns, W.R., Garrard, T.L., Israel, M.H., Klarmann, J., Stone, E.C., and Waddington, C.J., 1990. Determination of the Cross Sections for the Production of Fragments from Relativistic Nucleus-Nucleus Interactions I. Measurements. Phys. Rev. C 42, 2508-2529.

Fields, B.D., Olive, K.A., Schramm, D.N., 1994. Cosmic Ray Models for Early Galactic Lithium, Beryllium and Boron Production. Fermilab-Pub-94/010-A.

Flesch, F., Hirzebruch, S.E., Huntrup, G., Rocher, H., Streibel, T., Winkel, E., Heinrich, W., 1999. Fragmentation Cross Section Measurements of Iron Projectiles using CR-39 Plastic Nuclear Track Detectors. Radiat. Meas. 31, 533-536.

Garcis-Munoz, M., Simpson, J.A., Guzik, T.G., Wefel, J.P., Margolis, S.H., 1987, Cosmic-ray Propagation in the Galaxy and in the Heliosphere: The Path-Length Distribution at Low Energy. Astrophys. J. Suppl. 64, 269-304.

Hesse, A., et al., 1991. The Isotopic Composition of Silicon and Iron in the Cosmic Radiation as Measured by the ALICE Experiment. $22^{\text {nd }}$ International Conference, The Dublin Institute for Advanced Studies, Dublin, 596-599.

Iancu, G., Flesch, F., and Heinrich, W., 2005. Nuclear Fragmentation Cross-Sections of $400 \mathrm{~A} \mathrm{MeV} 36 \mathrm{Ar}$ and 40Ar in Collisions with Light and Heavy Target Nuclei. Radiat. Meas. 39, 525-533.

Kim, M., Wilson, J.W., Kiefer, R.L., Thibeault, S.A., 1994. Effects of Isotope Selection on Solution Convergence in HZE Transport. NASA TP-3445.

Knott, C.N., et al., 1996. Interactions of Relativistic Neon to Nickel Projectiles in Hydrogen, Elemental Production Cross-sections. Phys. Rev. C53, 347-357.

Knott, C.N., et al., 1997. Interactions of Relativistic ${ }^{36} \mathrm{Ar}$ and ${ }^{40} \mathrm{Ar}$ Nuclei in Hydrogen: Isotopic Production Cross-sections. Phys. Rev. C56, 398-406.

Letaw, J., Tsao, C.H., Silberberg, R., 1983. Matrix Methods of Cosmic Ray Propagation. 
Composition and Origin of Cosmic Rays, Maurice M. Shapiro, ed., D. Reidel Publ. Co, 337-342.

Lukasiak, A., Ferrando, P., McDonald, F.B., Webber, W.R., 1993. Cosmic Ray Composition of $6<\mathrm{Z}<8$ Nuclei in the Energy Range $50-150 \mathrm{MeV} / \mathrm{n}$ by the Voyager Spacecraft during the Solar Minimum and Maximum Periods. $23^{\text {rd }}$ International Cosmic Ray Conference, La Jolla, USA, 539-542.

Lukasiak, A., McDonald, F.B., Webber, W.R., Ferrando, P., 1995. Voyager Measurements of the Isotopic Composition of Sc, Ti, V, Cr, Mn, and Fe Nuclei. $24^{\text {th }}$ International Conference Ray Conference, Vol. 2, 576-579.

Norbury, J.W., Cucinotta, F.A., Townsend, L.W., Badavi, F.F., 1988. Parameterized Cross Sections in Heavy-Ion Collisions. Nucl. Instrum. Meth. Phys. Res. B31, 535-537.

Parker, E.N., 1965. The Passage of Energetic Charged Particles Through Interplanetary Space. Planet Space Sci. 13, 9-49.

Schimmerling, W., Miller, J., Wong, M., Rapkin, M., Howard, J., Spieler, H.G., Blair, J. V., 1989. The Fragmentation of 670A MeV Neon-20 as a Function of Depth in Water. Radiat. Res. 120, 36-51.

Shinn, J.L., Wilson, J.W., Badavi, F.F., 1994. Fully-Energy Dependent HZETRN. NASA TP-3243.

Tripathi, R.K., Wilson, J.W., Cucinotta, F.A., 2001. Medium Modified Two-Body Scattering Amplitude from Proton-Nucleus Total Cross-Sections. Nucl. Instrum. Meth. Phys. Res. B173, 391-396.

Webber, W.R., Kish, J.C., Schrier, D.A., 1985. Cosmic Ray Isotope Measurements with a New Cerenkov Total Energy Telescope. $19^{\text {th }}$ International Cosmic Ray Conference, La Jolla , USA, 88-95.

Webber, W.R., Southoul, A., Ferrando, P., Gupta, M., 1990a. The Source Charge and Isotropic Abundances of Cosmic Rays with $Z=9-16$ : A Study Using New Fragmentation Cross-Sections. The Astrophys. J. 348, 611-620.

Webber, W.R., Kish, J.C., Schrier, D.A., 1990b. Individual Isotopic Fragmentation Cross-Sections of Relativistic Nuclei in Hydrogen, Helium and Carbon Targets, Phys. Rev. C41, 547.

Webber, W.R., et al., 1998. Measurements of Charge Changing and Isotopic Cross Sections at $\sim 600 \mathrm{MeV} /$ nucleon from the Interactions of $\sim 30$ Separate Beams at Relativistic Nuclei from 10B to 55Mn in a Liquid Hydrogen Target. Phys. Rev. C 58, 3539-3552. 
Wiedenback, M.E., Greiner, D.E., 1981. High-Resolution Observations of the Isotopic Composition of Carbon And Silicon in the Galactic Cosmic Rays. The Astrophys. J. 247 L119-L122.

Wiedenback, M.E., 1985. The Isotopic Composition on Cosmic Ray Chlorine, $19^{\text {th }}$ International Cosmic Ray Conference, La Jolla , USA, 1985, 84-87.

Wilson, J.W., 1977. Analysis of Theory of High-Energy Transport. NASA TN D-8381.

Wilson, J.W., Badavi, F.F., 1986. Methods of Galactic Heavy Ion Transport. Radiat. Res. 108, 231-237.

Wilson, J.W., Townsend, L.W., Schimmerling, W., Khandelwal, G.S., Khan, F., Nealy, J. E., Cucinotta, F.A., Simonsen, L.C., Shinn, J.L., Norbury, J.W., 1991. Transport Methods and Interactions for Space Radiations. NASA RP-1257.

Wilson, J.W., Thibeault, S.A., Nealy, J.E., Kim, M.Y., Kiefer, R.L., 1993. Studies in Space Radiation Shield Performance. Proceedings of the Engineering \& Architecture Symposium, Prairie View A\&M Univ., 169-176.

Wilson, J.W., Shavers, M.R., Badavi, F.F., Miller, J., Shinn, J.L., Costen, R. C., 1994. Non-perturbative Methods in HZE Propagation. Radiat. Res. 140, 241-248.

Wilson, J.W., et al., 1995. NUCFRG2: An Evaluation of the Semi-empirical Nuclear Fragmentation Database. NASA TP-3533.

Yanasak, N.E., Binns, W.R., Cummings, A.C., Christian, E.R., George, J.S., Hink, P.L., Klarmann, J., Leske, R.A., Lijowski,M., Mewaldt, R.A., Stone, E.C., von Rosenvinge, T. T., Wiedenbeck, M.E., 1999. Implications for Cosmic Ray Propagation from ACE Measurements of Radioactive Clock Isotope Abundances, Proceedings of the $26^{\text {th }}$ International Cosmic Ray Conference, Vol. 3, 9.

Zeitlin, C., Heilbronn, L., Miller, J., Rademacher, S.E., Borak, T., Carter, T.R., Frankel, K.A., Schimmerling, W., Stronach, C.E., 1997. Heavy Fragment Production Crosssections from $1.05 \mathrm{GeV} /$ nucleon ${ }^{56} \mathrm{Fe}$ in $\mathrm{C}, \mathrm{Al}, \mathrm{Cu}, \mathrm{Pb}$, and $\mathrm{CH}_{2}$ Target. Phys. Rev. C56, 388-397.

Zeitlin, C., Fukumura, A., Heilbronn, L., Iwata, Y., Miller, J., Murakami, T., 2001. Fragmentation Cross-sections of $600 \mathrm{MeV} /$ nucleon ${ }^{20} \mathrm{Ne}$ on Elemental Targets. Phys. Rev. C64, 24902.

Zeitlin, C., Cleghorn, T., Cucinotta, F., Saganti, P., Andersen, V., Lee, K., Pinsky, L., Atwell, W., Turner, R., and Badhwar, G., 2004. Overview of the Martian Radiation Environment Experiment. Adv. Space Res. 33, 2204-2210. 
Table 1a: Isotopic Composition of GCR Elements $Z=3$ to 12 .

\begin{tabular}{|c|c|c|}
\hline Isotope & Near-Earth Fraction & Source Fraction \\
\hline \multicolumn{3}{|c|}{$Z=3$} \\
\hline${ }^{6} \mathrm{Li}^{*}$ & 0.5 & 0.5 \\
\hline${ }^{7} \mathrm{Li}$ & 0.5 & 0.5 \\
\hline \multicolumn{3}{|c|}{$Z=4$} \\
\hline${ }^{7} \mathrm{Be}^{*}$ & 0.5 & 0.5 \\
\hline${ }^{9} \mathrm{Be}$ & 0.35 & 0.35 \\
\hline${ }^{10} \mathrm{Be}$ & 0.15 & 0.15 \\
\hline \multicolumn{3}{|c|}{$Z=5$} \\
\hline${ }^{10} \mathrm{~B}$ & 0.31 & 0.2 \\
\hline${ }^{11} \mathrm{~B}$ & 0.69 & 0.8 \\
\hline \multicolumn{3}{|c|}{$Z=6$} \\
\hline${ }^{12} \mathrm{C}$ & 0.92 & 0.999 \\
\hline${ }^{13} \mathrm{C}$ & 0.08 & 0.001 \\
\hline \multicolumn{3}{|c|}{$Z=7$} \\
\hline${ }^{14} \mathrm{~N}$ & 0.48 & 0.78 \\
\hline${ }^{15} \mathrm{~N}$ & 0.57 & 0.22 \\
\hline \multicolumn{3}{|c|}{$Z=8$} \\
\hline${ }^{16} \mathrm{O}$ & 0.946 & 0.985 \\
\hline${ }^{17} \mathrm{O}$ & 0.027 & 0.008 \\
\hline${ }^{18} \mathrm{O}$ & 0.027 & 0.007 \\
\hline \multicolumn{3}{|c|}{$Z=10$} \\
\hline${ }^{20} \mathrm{Ne}$ & 0.55 & 0.68 \\
\hline${ }^{21} \mathrm{Ne}$ & 0.10 & 0.0 \\
\hline${ }^{22} \mathrm{Ne}$ & 0.35 & 0.32 \\
\hline \multicolumn{3}{|c|}{$Z=12$} \\
\hline${ }^{24} \mathrm{Mg}$ & 0.64 & 0.74 \\
\hline${ }^{25} \mathrm{Mg}$ & 0.18 & 0.14 \\
\hline${ }^{26} \mathrm{Mg}$ & 0.18 & 0.13 \\
\hline
\end{tabular}

*Data on solar modulation was not found and thus near-Earth and source composition are set equal. 
Table 1b: Isotopic Composition of GCR Elements $Z=13$ to 20 .

\begin{tabular}{|c|c|c|}
\hline Isotope & Near-Earth Fraction & Source Fraction \\
\hline \multicolumn{3}{|c|}{$Z=13$} \\
\hline${ }^{26} \mathrm{Al}$ & 0.02 & 0.0 \\
\hline${ }^{27} \mathrm{Al}$ & 0.98 & 1.0 \\
\hline \multicolumn{3}{|c|}{$Z=14$} \\
\hline${ }^{28} \mathrm{Si}$ & 0.84 & 0.902 \\
\hline${ }^{29} \mathrm{Si}$ & 0.08 & 0.054 \\
\hline${ }^{30} \mathrm{Si}$ & 0.08 & 0.044 \\
\hline \multicolumn{3}{|c|}{$Z=16$} \\
\hline${ }^{32} \mathrm{~S}$ & 0.69 & 0.96 \\
\hline${ }^{33} \mathrm{~S}$ & 0.15 & 0.02 \\
\hline${ }^{34} \mathrm{~S}$ & 0.16 & 0.02 \\
\hline \multicolumn{3}{|c|}{$Z=17$} \\
\hline${ }^{35} \mathrm{Cl}$ & 0.52 & 1.0 \\
\hline${ }^{36} \mathrm{Cl}$ & 0.41 & 0.0 \\
\hline${ }^{37} \mathrm{Cl}$ & 0.26 & 0.0 \\
\hline \multicolumn{3}{|c|}{$Z=18$} \\
\hline${ }^{36} \mathrm{Ar}$ & 0.64 & 1.0 \\
\hline${ }^{37} \mathrm{Ar}$ & 0.03 & 0.0 \\
\hline${ }^{38} \mathrm{Ar}$ & 0.30 & 0.0 \\
\hline${ }^{40} \mathrm{Ar}$ & 0.03 & 0.0 \\
\hline \multicolumn{3}{|c|}{$Z=20$} \\
\hline${ }^{40} \mathrm{Ca}$ & 0.4 & 1.0 \\
\hline${ }^{41} \mathrm{Ca}$ & 0.2 & 0.0 \\
\hline${ }^{42} \mathrm{Ca}$ & 0.2 & 0.0 \\
\hline${ }^{43} \mathrm{Ca}$ & 0.2 & 0.0 \\
\hline${ }^{44} \mathrm{Ca}$ & 0.2 & 0.0 \\
\hline
\end{tabular}


Table 1c: Isotopic Composition of GCR Elements $Z=22$ to 26.

\begin{tabular}{|c|c|c|}
\hline Isotope & Near-Earth Fraction & Source Fraction \\
\hline \multicolumn{3}{|c|}{$Z=22$} \\
\hline${ }^{46} \mathrm{Ti}^{*}$ & 0.27 & 0.27 \\
\hline${ }^{47} \mathrm{Ti}$ & 0.31 & 0.31 \\
\hline${ }^{48} \mathrm{Ti}$ & 0.30 & 0.30 \\
\hline${ }^{49} \mathrm{Ti}$ & 0.09 & 0.09 \\
\hline${ }^{50} \mathrm{Ti}$ & 0.03 & 0.03 \\
\hline \multicolumn{3}{|c|}{$Z=23$} \\
\hline${ }^{49} \mathrm{~V}^{*}$ & 0.53 & 0.53 \\
\hline${ }^{50} \mathrm{~V}$ & 0.24 & 0.24 \\
\hline${ }^{51} \mathrm{~V}$ & 0.23 & 0.23 \\
\hline \multicolumn{3}{|c|}{$Z=24$} \\
\hline${ }^{50} \mathrm{Cr}^{*}$ & 0.26 & 0.26 \\
\hline${ }^{51} \mathrm{Cr}$ & 0.26 & 0.26 \\
\hline${ }^{52} \mathrm{Cr}$ & 0.48 & 0.48 \\
\hline \multicolumn{3}{|c|}{$Z=25$} \\
\hline${ }^{53} \mathrm{Mn}^{*}$ & 0.43 & 0.43 \\
\hline${ }^{54} \mathrm{Mn}$ & 0.17 & 0.17 \\
\hline${ }^{55} \mathrm{Mn}$ & 0.40 & 0.40 \\
\hline \multicolumn{3}{|c|}{$Z=26$} \\
\hline${ }^{54} \mathrm{Fe}$ & 0.076 & 0.055 \\
\hline${ }^{55} \mathrm{Fe}$ & 0.084 & 0.078 \\
\hline${ }^{56} \mathrm{Fe}$ & 0.763 & 0.792 \\
\hline${ }^{57} \mathrm{Fe}$ & 0.076 & 0.075 \\
\hline
\end{tabular}

*Data on solar modulation was not found and thus near-Earth and source composition are set equal. 
Table 2a. Isotopic Grid Used in Present HZETRN Calculations for Elements $Z=0-11$.

\begin{tabular}{|c|c|c|c|c|c|c|c|c|c|}
\hline Index, $j$ & Nuclei & $T z$ & Lifetime & Decay & Index, $j$ & Nuclei & $T z$ & Lifetime & Decay \\
\hline 1 & ${ }^{1} n$ & $-1 / 2$ & $614.6 \mathrm{~s}$ & $\beta-$ & 24 & ${ }^{12} \mathrm{~N}$ & +1 & $0.0111 \mathrm{~s}$ & $\mathrm{e}+, \beta+$ \\
\hline 2 & ${ }^{1} \mathrm{H}$ & $+1 / 2$ & Stable & & 27 & ${ }^{13} \mathrm{~N}$ & $+1 / 2$ & $9.965 \mathrm{~m}$ & $\mathrm{e}+, \beta+$ \\
\hline 3 & ${ }^{2} \mathrm{H}$ & 0 & Stable & & 29 & ${ }^{14} \mathrm{~N}$ & 0 & Stable & \\
\hline 4 & ${ }^{3} \mathrm{H}$ & $-1 / 2$ & $12.33 \mathrm{y}$ & $\beta-$ & 32 & ${ }^{15} \mathrm{~N}$ & $-1 / 2$ & Stable & \\
\hline 5 & ${ }^{3} \mathrm{He}$ & $+1 / 2$ & Stable & & 34 & ${ }^{16} \mathrm{~N}$ & -1 & $7.13 \mathrm{~s}$ & $\beta-$ \\
\hline 6 & ${ }^{4} \mathrm{He}$ & 0 & Stable & & 36 & ${ }^{17} \mathrm{~N}$ & $-3 / 2$ & $4.173 \mathrm{~s}$ & $\beta-$ \\
\hline 7 & ${ }^{6} \mathrm{He}$ & -1 & $0.807 \mathrm{~s}$ & $\beta-$ & 30 & ${ }^{14} \mathrm{O}$ & +1 & $70.606 \mathrm{~s}$ & $\mathrm{e}+, \beta+$ \\
\hline 8 & ${ }^{6} \mathrm{Li}$ & 0 & Stable & & 33 & ${ }^{15} \mathrm{O}$ & $+1 / 2$ & $122.24 \mathrm{~s}$ & $e+, \beta+$ \\
\hline 9 & ${ }^{7} \mathrm{Li}$ & $-1 / 2$ & Stable & & 35 & ${ }^{16} \mathrm{O}$ & 0 & Stable & \\
\hline 11 & ${ }^{8} \mathrm{Li}$ & -1 & $0.838 \mathrm{~s}$ & $\beta-$ & 37 & ${ }^{17} \mathrm{O}$ & $-1 / 2$ & Stable & \\
\hline 13 & ${ }^{9} \mathrm{Li}$ & $-3 / 2$ & $0.178 \mathrm{~s}$ & $\beta-$ & 39 & ${ }^{18} \mathrm{O}$ & -1 & Stable & \\
\hline 10 & ${ }^{7} \mathrm{Be}$ & $+1 / 2$ & $53.12 d$ & e & 42 & ${ }^{19} \mathrm{O}$ & $-3 / 2$ & $26.91 \mathrm{~s}$ & $\beta-$ \\
\hline 14 & ${ }^{9} \mathrm{Be}$ & $-1 / 2$ & Stable & & 45 & ${ }^{20} \mathrm{O}$ & -2 & $13.51 \mathrm{~s}$ & $\beta-$ \\
\hline 16 & ${ }^{10} \mathrm{Be}$ & -1 & $1.51 \times 10^{6} \mathrm{y}$ & $\beta-$ & 38 & ${ }^{17} \mathrm{~F}$ & $+1 / 2$ & $64.49 \mathrm{~s}$ & $\mathrm{e}+, \beta+$ \\
\hline 19 & ${ }^{11} \mathrm{Be}$ & $-3 / 2$ & $13.81 \mathrm{~s}$ & $\beta-$ & 40 & ${ }^{18} \mathrm{~F}$ & 0 & $0.1098 \mathrm{~s}$ & $e+\beta+$ \\
\hline 12 & ${ }^{8} \mathrm{~B}$ & +1 & $0.770 \mathrm{~s}$ & $\beta+$ & 43 & ${ }^{19} \mathrm{~F}$ & $-1 / 2$ & Stable & \\
\hline 15 & ${ }^{9} \mathrm{~B}$ & $+1 / 2$ & $0.54 \mathrm{keV}$ & $2 \alpha p$ & 46 & ${ }^{20} \mathrm{~F}$ & -1 & $11.0 \mathrm{~s}$ & $\beta-$ \\
\hline 17 & ${ }^{10} \mathrm{~B}$ & 0 & Stable & & 48 & ${ }^{21} \mathrm{~F}$ & $-3 / 2$ & $4.158 \mathrm{~s}$ & $\beta-$ \\
\hline 20 & ${ }^{11} \mathrm{~B}$ & $-1 / 2$ & Stable & & 41 & ${ }^{18} \mathrm{Ne}$ & +1 & $1.672 \mathrm{~s}$ & $\mathrm{e}+, \beta+$ \\
\hline 22 & ${ }^{12} \mathrm{~B}$ & -1 & $0.0202 \mathrm{~s}$ & $\beta-$ & 44 & ${ }^{19} \mathrm{Ne}$ & $+1 / 2$ & $17.22 \mathrm{~s}$ & $\mathrm{e}+, \beta+$ \\
\hline 25 & ${ }^{13} \mathrm{~B}$ & $-3 / 2$ & $0.01736 \mathrm{~s}$ & $\beta-$ & 47 & ${ }^{20} \mathrm{Ne}$ & 0 & Stable & \\
\hline 18 & ${ }^{10} \mathrm{C}$ & +1 & $19.255 \mathrm{~s}$ & $e+, \beta+$ & 49 & ${ }^{21} \mathrm{Ne}$ & $-1 / 2$ & Stable & \\
\hline 21 & ${ }^{11} \mathrm{C}$ & $+1 / 2$ & $0.0204 \mathrm{~s}$ & $\mathrm{e}+, \beta+$ & 51 & ${ }^{22} \mathrm{Ne}$ & -1 & Stable & \\
\hline 23 & ${ }^{12} \mathrm{C}$ & 0 & Stable & & 53 & ${ }^{23} \mathrm{Ne}$ & $-3 / 2$ & $37.24 \mathrm{~s}$ & $\beta-$ \\
\hline 26 & ${ }^{13} \mathrm{C}$ & $-1 / 2$ & Stable & & 56 & ${ }^{24} \mathrm{Ne}$ & -2 & $3.38 \mathrm{~m}$ & $\beta-$ \\
\hline 28 & ${ }^{14} \mathrm{C}$ & -1 & $5730 y$ & $\beta-$ & 50 & ${ }^{21} \mathrm{Na}$ & $+1 / 2$ & $22.49 \mathrm{~s}$ & $\mathrm{e}+, \beta+$ \\
\hline \multirow[t]{5}{*}{31} & ${ }^{15} \mathrm{C}$ & $-3 / 2$ & $2.449 \mathrm{~s}$ & $\beta-$ & 52 & ${ }^{22} \mathrm{Na}$ & 0 & $2.6019 y$ & $\mathrm{e}+, \beta+$ \\
\hline & & & & & 54 & ${ }^{23} \mathrm{Na}$ & $-1 / 2$ & stable & \\
\hline & & & & & 57 & ${ }^{24} \mathrm{Na}$ & -1 & $14.959 \mathrm{~h}$ & $\beta-$ \\
\hline & & & & & 59 & ${ }^{25} \mathrm{Na}$ & $-3 / 2$ & $59.1 \mathrm{~s}$ & $\beta-$ \\
\hline & & & & & 62 & ${ }^{26} \mathrm{Na}$ & -2 & $1.072 \mathrm{~s}$ & $\beta-$ \\
\hline
\end{tabular}


Table 2b. Isotopic Grid Used in Present HZETRN Calculations for Elements Z=12-18.

\begin{tabular}{|c|c|c|c|c|c|c|c|c|c|}
\hline Index, $j$ & Nuclei & $T z$ & Lifetime & Decay & Index, $j$ & Nuclei & $T z$ & Lifetime & Decay \\
\hline 55 & ${ }^{23} \mathrm{Mg}$ & $+1 / 2$ & $11.317 \mathrm{~s}$ & $e+, \beta+$ & 78 & ${ }^{30} \mathrm{~S}$ & +1 & $1.178 \mathrm{~s}$ & $\mathrm{e}+, \beta+$ \\
\hline 58 & ${ }^{24} \mathrm{Mg}$ & 0 & stable & & 81 & ${ }^{31} \mathrm{~S}$ & $+1 / 2$ & $2.572 \mathrm{~s}$ & $\mathrm{e}+, \beta+$ \\
\hline 60 & ${ }^{25} \mathrm{Mg}$ & $-1 / 2$ & stable & & 84 & ${ }^{32} \mathrm{~S}$ & 0 & Stable & \\
\hline 63 & ${ }^{26} \mathrm{Mg}$ & -1 & stable & & 87 & ${ }^{33} \mathrm{~S}$ & $-1 / 2$ & Stable & \\
\hline 66 & ${ }^{27} \mathrm{Mg}$ & $-3 / 2$ & $9.458 \mathrm{~m}$ & $\beta-$ & 90 & ${ }^{34} \mathrm{~S}$ & -1 & Stable & \\
\hline 69 & ${ }^{28} \mathrm{Mg}$ & -2 & $20.91 \mathrm{~h}$ & $\beta-$ & 93 & ${ }^{35} \mathrm{~S}$ & $-3 / 2$ & $87.32 \mathrm{~d}$ & $\beta-$ \\
\hline 61 & ${ }^{25} \mathrm{Al}$ & $+1 / 2$ & $7.183 \mathrm{~s}$ & $e+, \beta+$ & 96 & ${ }^{36} \mathrm{~S}$ & -2 & Stable & \\
\hline 64 & ${ }^{26} \mathrm{Al}$ & 0 & $7.17 \times 10^{5} \mathrm{y}$ & $\mathrm{e}+, \beta+$ & 99 & ${ }^{37} \mathrm{~S}$ & $-5 / 2$ & $5.05 \mathrm{~m}$ & $\beta-$ \\
\hline 67 & ${ }^{27} \mathrm{Al}$ & $-1 / 2$ & stable & & 103 & ${ }^{38} \mathrm{~S}$ & -2 & $170.3 \mathrm{~m}$ & $\beta-$ \\
\hline 70 & ${ }^{28} \mathrm{Al}$ & -1 & $2.241 \mathrm{~m}$ & $\beta-$ & 88 & ${ }^{33} \mathrm{Cl}$ & $+1 / 2$ & $2.511 \mathrm{~s}$ & $\mathrm{e}+, \beta+$ \\
\hline 72 & ${ }^{29} \mathrm{Al}$ & $-3 / 2$ & $6.56 \mathrm{~m}$ & $\beta-$ & 91 & ${ }^{34} \mathrm{Cl}$ & 0 & $1.5264 \mathrm{~s}$ & $\mathrm{e}+, \beta+$ \\
\hline 75 & ${ }^{30} \mathrm{Al}$ & -2 & $3.60 \mathrm{~s}$ & $\beta-$ & 94 & ${ }^{35} \mathrm{Cl}$ & $-1 / 2$ & Stable & \\
\hline 65 & ${ }^{26} \mathrm{Si}$ & +1 & $2.234 \mathrm{~s}$ & $e+, \beta+$ & 97 & ${ }^{36} \mathrm{Cl}$ & -1 & $3.01 \times 10^{5} \mathrm{y}$ & $\beta-$ \\
\hline 68 & ${ }^{27} \mathrm{Si}$ & $+1 / 2$ & $4.16 \mathrm{~s}$ & $\mathrm{e}+, \beta+$ & 100 & ${ }^{37} \mathrm{Cl}$ & $-3 / 2$ & Stable & \\
\hline 71 & ${ }^{28} \mathrm{Si}$ & 0 & stable & & 104 & ${ }^{38} \mathrm{Cl}$ & -2 & $37.34 \mathrm{~m}$ & $\beta-$ \\
\hline 73 & ${ }^{29} \mathrm{Si}$ & $-1 / 2$ & stable & & 108 & ${ }^{39} \mathrm{Cl}$ & $-5 / 2$ & $55.60 \mathrm{~m}$ & $\beta-$ \\
\hline 76 & ${ }^{30} \mathrm{Si}$ & -1 & stable & & 95 & ${ }^{35} \mathrm{Ar}$ & $+1 / 2$ & $1.775 \mathrm{~s}$ & $e+, \beta+$ \\
\hline 79 & ${ }^{31} \mathrm{Si}$ & $-3 / 2$ & $0.1573 \mathrm{~s}$ & $\beta-$ & 98 & ${ }^{36} \mathrm{Ar}$ & 0 & Stable & \\
\hline 82 & ${ }^{32} \mathrm{Si}$ & -2 & $150 y$ & $\beta-$ & 101 & ${ }^{37} \mathrm{Ar}$ & $-1 / 2$ & $35.04 \mathrm{~d}$ & e \\
\hline 85 & ${ }^{33} \mathrm{Si}$ & $-5 / 2$ & $6.18 \mathrm{~s}$ & $\beta-$ & 105 & ${ }^{38} \mathrm{Ar}$ & -1 & Stable & \\
\hline 74 & ${ }^{29} \mathrm{P}$ & $+1 / 2$ & $4.140 \mathrm{~s}$ & $e+, \beta+$ & 109 & ${ }^{39} \mathrm{Ar}$ & $-3 / 2$ & 269 y & $\beta-$ \\
\hline 77 & ${ }^{30} \mathrm{P}$ & 0 & $2.498 \mathrm{~m}$ & $\mathrm{e}+, \beta+$ & 112 & ${ }^{40} \mathrm{Ar}$ & -2 & Stable & \\
\hline 80 & ${ }^{31} \mathrm{P}$ & $-1 / 2$ & stable & & 115 & ${ }^{41} \mathrm{Ar}$ & $-5 / 2$ & $109.34 \mathrm{~m}$ & $\beta-$ \\
\hline 83 & ${ }^{32} \mathrm{P}$ & -1 & $14.262 \mathrm{~d}$ & $\beta-$ & 118 & ${ }^{42} \mathrm{Ar}$ & -3 & 32.9 y & $\beta-$ \\
\hline 86 & ${ }^{33} \mathrm{P}$ & $-3 / 2$ & $25.34 d$ & $\beta-$ & & & & & \\
\hline 89 & ${ }^{34} \mathrm{P}$ & -2 & $12.43 \mathrm{~s}$ & $\beta-$ & & & & & \\
\hline 92 & ${ }^{35} \mathrm{P}$ & $-5 / 2$ & $47.3 \mathrm{~s}$ & $\beta-$ & & & & & \\
\hline
\end{tabular}


Table 2c. Isotopic Grid Used in Present HZETRN Calculations for Elements Z=19-28.

\begin{tabular}{|c|c|c|c|c|c|c|c|c|c|}
\hline Index, $j$ & Nuclei & $T z$ & Lifetime & Decay & Index, $j$ & Nuclei & $T z$ & Lifetime & Decay \\
\hline 102 & ${ }^{3 /} \mathrm{K}$ & $+1 / 2$ & $1.226 \mathrm{~s}$ & $e+, \beta+$ & 134 & ${ }^{46} \mathrm{~V}$ & 0 & $0.422 \mathrm{~s}$ & $\mathrm{e}+, \beta+$ \\
\hline 106 & ${ }^{38} \mathrm{~K}$ & 0 & $7.636 \mathrm{~m}$ & $\mathrm{e}+, \beta+$ & 137 & ${ }^{47} \mathrm{~V}$ & $-1 / 2$ & $32.6 \mathrm{~m}$ & $\mathrm{e}+, \beta+$ \\
\hline 110 & ${ }^{39} \mathrm{~K}$ & $-1 / 2$ & Stable & & 140 & ${ }^{48} \mathrm{~V}$ & -1 & $15.9735 \mathrm{~d}$ & $\mathrm{e}+, \beta+$ \\
\hline 113 & ${ }^{40} \mathrm{~K}$ & -1 & $1.277 \times 10^{9} \mathrm{y}$ & $\beta-$ & 143 & ${ }^{49} \mathrm{~V}$ & $-3 / 2$ & $330 \mathrm{~d}$ & $\mathrm{e}$ \\
\hline 116 & ${ }^{41} \mathrm{~K}$ & $-3 / 2$ & Stable & & 146 & ${ }^{50} \mathrm{~V}$ & -2 & $1.4 \times 10^{17} \mathrm{y}$ & $\mathrm{e}+, \beta+$ \\
\hline 119 & ${ }^{42} \mathrm{~K}$ & -2 & $12.36 \mathrm{~h}$ & $\beta-$ & 149 & ${ }^{51} \mathrm{~V}$ & $-5 / 2$ & Stable & \\
\hline 122 & ${ }^{43} \mathrm{~K}$ & $-5 / 2$ & $22.3 \mathrm{~h}$ & $\beta-$ & 152 & ${ }^{52} \mathrm{~V}$ & -3 & $3.743 \mathrm{~m}$ & $\beta-$ \\
\hline 107 & ${ }^{38} \mathrm{Ca}$ & +1 & $0.440 \mathrm{~s}$ & $e+, \beta+$ & 141 & ${ }^{48} \mathrm{Cr}$ & 0 & $21.56 \mathrm{~h}$ & $\mathrm{e}+, \beta+$ \\
\hline 111 & ${ }^{39} \mathrm{Ca}$ & $+1 / 2$ & $0.8596 \mathrm{~s}$ & $e+, \beta+$ & 144 & ${ }^{49} \mathrm{Cr}$ & $-1 / 2$ & $42.3 \mathrm{~m}$ & $\mathrm{e}+, \beta+$ \\
\hline 114 & ${ }^{40} \mathrm{Ca}$ & 0 & Stable & & 147 & ${ }^{50} \mathrm{Cr}$ & -1 & $>1.8 \times 10^{17} y$ & $\mathrm{e}$ \\
\hline 117 & ${ }^{41} \mathrm{Ca}$ & $-1 / 2$ & $1.03 \times 10^{5} \mathrm{y}$ & $\mathrm{e}$ & 150 & ${ }^{51} \mathrm{Cr}$ & $-3 / 2$ & $27.7025 \mathrm{~d}$ & $\mathrm{e}$ \\
\hline 120 & ${ }^{42} \mathrm{Ca}$ & -1 & Stable & & 153 & ${ }^{52} \mathrm{Cr}$ & -2 & Stable & \\
\hline 123 & ${ }^{43} \mathrm{Ca}$ & $-3 / 2$ & Stable & & 155 & ${ }^{53} \mathrm{Cr}$ & $-5 / 2$ & Stable & \\
\hline 125 & ${ }^{44} \mathrm{Ca}$ & -2 & Stable & & 158 & ${ }^{54} \mathrm{Cr}$ & -3 & Stable & \\
\hline 128 & ${ }^{45} \mathrm{Ca}$ & $-5 / 2$ & $162.61 \mathrm{~d}$ & $\beta-$ & 148 & ${ }^{50} \mathrm{Mn}$ & 0 & $0.284 \mathrm{~s}$ & $e+, \beta+$ \\
\hline 131 & ${ }^{46} \mathrm{Ca}$ & -3 & Stable & & 151 & ${ }^{51} \mathrm{Mn}$ & $-1 / 2$ & $0.0462 \mathrm{~s}$ & $e+, \beta+$ \\
\hline 121 & ${ }^{42} \mathrm{Sc}$ & 0 & $0.681 \mathrm{~s}$ & $\mathrm{e}+, \beta+$ & 154 & ${ }^{52} \mathrm{Mn}$ & -1 & $5.591 \mathrm{~d}$ & $\mathrm{e}+, \beta+$ \\
\hline 124 & ${ }^{43} \mathrm{Sc}$ & $-1 / 2$ & $3.891 \mathrm{~h}$ & $\mathrm{e}+, \beta+$ & 156 & ${ }^{53} \mathrm{Mn}$ & $-3 / 2$ & $3.74 \times 10^{6}$ & e \\
\hline 126 & ${ }^{44} \mathrm{Sc}$ & -1 & $3.927 \mathrm{~h}$ & $\mathrm{e}+, \beta+$ & 159 & ${ }^{54} \mathrm{Mn}$ & -2 & $312.3 \mathrm{~d}$ & $\mathrm{e}+, \beta+$ \\
\hline 129 & ${ }^{45} \mathrm{Sc}$ & $-3 / 2$ & stable & & 161 & ${ }^{55} \mathrm{Mn}$ & $-5 / 2$ & Stable & \\
\hline 132 & ${ }^{46} \mathrm{Sc}$ & -2 & $83.79 \mathrm{~d}$ & $\beta-$ & 157 & ${ }^{53} \mathrm{Fe}$ & $-1 / 2$ & $0.00851 \mathrm{~s}$ & $e+, \beta+$ \\
\hline 135 & ${ }^{47} \mathrm{Sc}$ & $-5 / 2$ & $3.3492 \mathrm{~d}$ & $\beta-$ & 160 & ${ }^{54} \mathrm{Fe}$ & -1 & Stable & \\
\hline 138 & ${ }^{48} \mathrm{Sc}$ & -3 & $43.67 \mathrm{~h}$ & $\beta-$ & 162 & ${ }^{55} \mathrm{Fe}$ & $-3 / 2$ & $2.73 \mathrm{y}$ & e \\
\hline 127 & ${ }^{44} \mathrm{Ti}$ & 0 & $63 y$ & e & 164 & ${ }^{56} \mathrm{Fe}$ & -2 & Stable & \\
\hline 130 & ${ }^{45} \mathrm{Ti}$ & $-1 / 2$ & $184.8 \mathrm{~m}$ & $e+, \beta+$ & 167 & ${ }^{57} \mathrm{Fe}$ & $-5 / 2$ & Stable & \\
\hline 133 & ${ }^{46} \mathrm{Ti}$ & -1 & Stable & & 163 & ${ }^{55} \mathrm{Co}$ & $-1 / 2$ & $17.53 \mathrm{~h}$ & $e+, \beta+$ \\
\hline 136 & ${ }^{47} \mathrm{Ti}$ & $-3 / 2$ & Stable & & 165 & ${ }^{56} \mathrm{Co}$ & -1 & $77.27 \mathrm{~d}$ & $e+, \beta+$ \\
\hline 139 & ${ }^{48} \mathrm{Ti}$ & -2 & Stable & & 168 & ${ }^{57} \mathrm{Co}$ & $-3 / 2$ & $271.79 \mathrm{~d}$ & $\mathrm{e}$ \\
\hline 142 & ${ }^{49} \mathrm{Ti}$ & $-5 / 2$ & Stable & & 166 & ${ }^{56} \mathrm{Ni}$ & 0 & $6.077 \mathrm{~d}$ & $\mathrm{e}+, \beta+$ \\
\hline \multirow[t]{2}{*}{145} & ${ }^{50} \mathrm{Ti}$ & -3 & Stable & & 169 & ${ }^{57} \mathrm{Ni}$ & $-1 / 2$ & $35.60 \mathrm{~h}$ & $\mathrm{e}+, \beta+$ \\
\hline & & & & & 170 & ${ }^{58} \mathrm{Ni}$ & -1 & Stable & \\
\hline
\end{tabular}


Table 3a: Elemental (Z) and Neutron excess (Y) dependence on GCR dose equivalent behind $0 \mathrm{~g} / \mathrm{cm}^{2}$ of Aluminum Shielding.

\begin{tabular}{|c|c|c|c|c|c|c|c|}
\hline \multicolumn{8}{|c|}{ GCR Dose Equivalent per Year for near Solar Minimum } \\
\hline Z & $\mathrm{Y}<0$ & $Y=0$ & $\mathrm{Y}=1$ & $\mathrm{Y}=2$ & $\mathrm{Y}=3$ & $Y>3$ & Total-Z \\
\hline 0 & 0.00 & 0.00 & 0.00 & 0.00 & 0.00 & 0.00 & 0.00 \\
\hline 1 & 9.15 & 0.21 & 0.00 & 0.00 & 0.00 & 0.00 & 9.37 \\
\hline 2 & 0.50 & 3.61 & 0.00 & 0.00 & 0.00 & 0.00 & 4.11 \\
\hline 3 & 0.00 & 0.04 & 0.04 & 0.00 & 0.00 & 0.00 & 0.08 \\
\hline 4 & 0.05 & 0.00 & 0.03 & 0.01 & 0.00 & 0.00 & 0.10 \\
\hline 5 & 0.00 & 0.23 & 0.36 & 0.00 & 0.00 & 0.00 & 0.60 \\
\hline 6 & 0.00 & 3.72 & 0.31 & 0.00 & 0.00 & 0.00 & 4.03 \\
\hline 7 & 0.00 & 0.74 & 0.99 & 0.00 & 0.00 & 0.00 & 1.73 \\
\hline 8 & 0.00 & 11.26 & 0.32 & 0.32 & 0.00 & 0.00 & 11.90 \\
\hline 9 & 0.00 & 0.00 & 0.38 & 0.00 & 0.00 & 0.00 & 0.38 \\
\hline 10 & 0.00 & 2.33 & 0.45 & 1.48 & 0.00 & 0.00 & 4.26 \\
\hline 11 & 0.00 & 0.00 & 1.33 & 0.00 & 0.00 & 0.00 & 1.33 \\
\hline 12 & 0.00 & 6.89 & 1.86 & 1.88 & 0.00 & 0.00 & 10.63 \\
\hline 13 & 0.00 & 0.12 & 2.34 & 0.00 & 0.00 & 0.00 & 2.46 \\
\hline 14 & 0.00 & 11.22 & 1.08 & 1.06 & 0.00 & 0.00 & 13.36 \\
\hline 15 & 0.00 & 0.00 & 0.85 & 0.00 & 0.00 & 0.00 & 0.85 \\
\hline 16 & 0.00 & 2.87 & 0.59 & 0.65 & 0.00 & 0.00 & 4.11 \\
\hline 17 & 0.00 & 0.00 & 0.52 & 0.41 & 0.26 & 0.00 & 1.19 \\
\hline 18 & 0.00 & 1.37 & 0.06 & 0.68 & 0.00 & 0.05 & 2.15 \\
\hline 19 & 0.00 & 0.00 & 1.71 & 0.00 & 0.00 & 0.00 & 1.71 \\
\hline 20 & 0.00 & 1.57 & 0.00 & 0.78 & 0.78 & 0.78 & 3.92 \\
\hline 21 & 0.00 & 0.00 & 0.00 & 0.00 & 1.00 & 0.00 & 1.00 \\
\hline 22 & 0.00 & 0.00 & 0.00 & 1.02 & 1.17 & 1.59 & 3.78 \\
\hline 23 & 0.00 & 0.00 & 0.00 & 0.00 & 1.00 & 0.89 & 1.88 \\
\hline 24 & 0.00 & 0.00 & 0.00 & 1.02 & 1.02 & 1.89 & 3.93 \\
\hline 25 & 0.00 & 0.00 & 0.00 & 0.00 & 1.26 & 1.67 & 2.92 \\
\hline 26 & 0.00 & 0.00 & 0.00 & 2.05 & 2.26 & 22.50 & 26.80 \\
\hline 27 & 0.00 & 0.00 & 0.00 & 0.00 & 0.10 & 0.00 & 0.10 \\
\hline 28 & 0.00 & 0.00 & 0.00 & 1.45 & 0.00 & 0.00 & 1.45 \\
\hline tal-Y & 9.70 & 46.18 & 13.22 & 12.81 & 8.85 & 29.35 & 120.11 \\
\hline
\end{tabular}


Table 3b: Elemental $(\mathrm{Z})$ and Neutron excess (Y) dependence on GCR dose equivalent behind $5 \mathrm{~g} / \mathrm{cm}^{2}$ of Aluminum Shielding.

\begin{tabular}{|c|c|c|c|c|c|c|c|}
\hline \multicolumn{8}{|c|}{ GCR Dose Equivalent per Year near Solar Minimum } \\
\hline Z & $Y<0$ & $\mathrm{Y}=0$ & $\mathrm{Y}=1$ & $\mathrm{Y}=2$ & $\mathrm{Y}=3$ & $Y>3$ & Total-Z \\
\hline 0 & 0.00 & 0.00 & 0.00 & 0.65 & 0.00 & 0.00 & 0.65 \\
\hline 1 & 15.03 & 2.56 & 1.74 & 0.00 & 0.00 & 0.00 & 19.33 \\
\hline 2 & 2.48 & 15.60 & 0.00 & 0.00 & 0.00 & 0.00 & 18.08 \\
\hline 3 & 0.00 & 0.04 & 0.04 & 0.00 & 0.00 & 0.00 & 0.09 \\
\hline 4 & 0.05 & 0.00 & 0.04 & 0.02 & 0.00 & 0.00 & 0.12 \\
\hline 5 & 0.00 & 0.23 & 0.34 & 0.00 & 0.00 & 0.00 & 0.58 \\
\hline 6 & 0.03 & 3.14 & 0.29 & 0.01 & 0.00 & 0.00 & 3.47 \\
\hline 7 & 0.01 & 0.62 & 0.83 & 0.01 & 0.00 & 0.00 & 1.48 \\
\hline 8 & 0.07 & 8.67 & 0.27 & 0.27 & 0.00 & 0.00 & 9.28 \\
\hline 9 & 0.01 & 0.03 & 0.31 & 0.01 & 0.01 & 0.00 & 0.37 \\
\hline 10 & 0.03 & 1.69 & 0.37 & 1.10 & 0.01 & 0.00 & 3.20 \\
\hline 11 & 0.01 & 0.06 & 0.99 & 0.04 & 0.01 & 0.00 & 1.11 \\
\hline 12 & 0.05 & 4.71 & 1.33 & 1.34 & 0.01 & 0.00 & 7.44 \\
\hline 13 & 0.01 & 0.15 & 1.66 & 0.03 & 0.01 & 0.00 & 1.87 \\
\hline 14 & 0.06 & 7.46 & 0.77 & 0.75 & 0.01 & 0.00 & 9.06 \\
\hline 15 & 0.00 & 0.02 & 0.60 & 0.04 & 0.02 & 0.01 & 0.70 \\
\hline 16 & 0.02 & 1.86 & 0.42 & 0.48 & 0.02 & 0.01 & 2.82 \\
\hline 17 & 0.00 & 0.02 & 0.38 & 0.32 & 0.21 & 0.01 & 0.93 \\
\hline 18 & 0.01 & 0.89 & 0.08 & 0.51 & 0.04 & 0.06 & 1.59 \\
\hline 19 & 0.00 & 0.02 & 1.12 & 0.04 & 0.04 & 0.04 & 1.27 \\
\hline 20 & 0.01 & 1.03 & 0.03 & 0.58 & 0.57 & 0.58 & 2.81 \\
\hline 21 & 0.00 & 0.00 & 0.02 & 0.05 & 0.70 & 0.08 & 0.85 \\
\hline 22 & 0.00 & 0.01 & 0.03 & 0.72 & 0.81 & 1.12 & 2.69 \\
\hline 23 & 0.00 & 0.00 & 0.02 & 0.05 & 0.71 & 0.66 & 1.44 \\
\hline 24 & 0.00 & 0.00 & 0.03 & 0.68 & 0.73 & 1.33 & 2.77 \\
\hline 25 & 0.00 & 0.00 & 0.02 & 0.05 & 0.88 & 1.23 & 2.18 \\
\hline 26 & 0.00 & 0.00 & 0.03 & 1.34 & 1.50 & 13.90 & 16.77 \\
\hline 27 & 0.00 & 0.00 & 0.00 & 0.01 & 0.06 & 0.00 & 0.08 \\
\hline 28 & 0.00 & 0.00 & 0.01 & 0.87 & 0.00 & 0.00 & 0.88 \\
\hline tal-Y & 17.88 & 48.84 & 11.80 & 9.95 & 6.37 & 19.03 & 113.88 \\
\hline
\end{tabular}


Table 3c: Elemental (Z) and Neutron excess (Y) dependence on GCR dose equivalent behind $20 \mathrm{~g} / \mathrm{cm}^{2}$ of Aluminum Shielding.

\begin{tabular}{|c|c|c|c|c|c|c|c|}
\hline \multicolumn{8}{|c|}{ GCR Dose Equivalent per Year near Solar Minimum } \\
\hline Z & $Y<0$ & $Y=0$ & $\mathrm{Y}=1$ & $Y=2$ & $Y=3$ & $Y>3$ & Total-Z \\
\hline 0 & 0.00 & 0.00 & 0.00 & 2.00 & 0.00 & 0.00 & 2.00 \\
\hline 1 & 18.95 & 3.09 & 2.21 & 0.00 & 0.00 & 0.00 & 24.24 \\
\hline 2 & 2.67 & 17.03 & 0.00 & 0.00 & 0.00 & 0.00 & 19.69 \\
\hline 3 & 0.00 & 0.03 & 0.03 & 0.00 & 0.00 & 0.00 & 0.07 \\
\hline 4 & 0.04 & 0.00 & 0.04 & 0.02 & 0.00 & 0.00 & 0.10 \\
\hline 5 & 0.00 & 0.13 & 0.21 & 0.00 & 0.00 & 0.00 & 0.35 \\
\hline 6 & 0.05 & 1.51 & 0.13 & 0.02 & 0.00 & 0.00 & 1.72 \\
\hline 7 & 0.02 & 0.41 & 0.38 & 0.01 & 0.00 & 0.00 & 0.83 \\
\hline 8 & 0.11 & 3.69 & 0.12 & 0.11 & 0.00 & 0.00 & 4.02 \\
\hline 9 & 0.01 & 0.04 & 0.17 & 0.02 & 0.01 & 0.00 & 0.26 \\
\hline 10 & 0.04 & 0.75 & 0.16 & 0.46 & 0.01 & 0.00 & 1.43 \\
\hline 11 & 0.01 & 0.09 & 0.45 & 0.05 & 0.02 & 0.00 & 0.62 \\
\hline 12 & 0.08 & 1.84 & 0.52 & 0.50 & 0.01 & 0.00 & 2.95 \\
\hline 13 & 0.02 & 0.12 & 0.69 & 0.04 & 0.02 & 0.00 & 0.89 \\
\hline 14 & 0.08 & 2.57 & 0.29 & 0.26 & 0.02 & 0.01 & 3.22 \\
\hline 15 & 0.01 & 0.04 & 0.26 & 0.05 & 0.03 & 0.01 & 0.39 \\
\hline 16 & 0.03 & 0.71 & 0.15 & 0.18 & 0.03 & 0.02 & 1.12 \\
\hline 17 & 0.00 & 0.02 & 0.19 & 0.12 & 0.08 & 0.02 & 0.44 \\
\hline 18 & 0.01 & 0.34 & 0.07 & 0.19 & 0.05 & 0.04 & 0.70 \\
\hline 19 & 0.00 & 0.03 & 0.39 & 0.06 & 0.05 & 0.05 & 0.58 \\
\hline 20 & 0.02 & 0.47 & 0.05 & 0.20 & 0.19 & 0.19 & 1.11 \\
\hline 21 & 0.00 & 0.01 & 0.03 & 0.07 & 0.29 & 0.10 & 0.49 \\
\hline 22 & 0.00 & 0.01 & 0.04 & 0.30 & 0.33 & 0.46 & 1.14 \\
\hline 23 & 0.00 & 0.00 & 0.03 & 0.06 & 0.30 & 0.31 & 0.71 \\
\hline 24 & 0.00 & 0.01 & 0.04 & 0.26 & 0.31 & 0.55 & 1.16 \\
\hline 25 & 0.00 & 0.00 & 0.02 & 0.06 & 0.36 & 0.55 & 1.00 \\
\hline 26 & 0.00 & 0.00 & 0.03 & 0.43 & 0.53 & 4.13 & 5.13 \\
\hline 27 & 0.00 & 0.00 & 0.00 & 0.01 & 0.02 & 0.00 & 0.04 \\
\hline 28 & 0.00 & 0.00 & 0.01 & 0.24 & 0.00 & 0.00 & 0.25 \\
\hline otal-Y & 22.15 & 32.94 & 6.69 & 5.73 & 2.68 & 6.45 & 76.64 \\
\hline
\end{tabular}


Table 4: Annual fluence of several unstable GCR nuclei versus depth of aluminum shielding near solar minimum ( $\Phi=428 \mathrm{MV})$.

\begin{tabular}{|c|c|c|c|c|}
\hline & \multicolumn{4}{|c|}{ Fluence per $\mathrm{cm}^{2}$ per year } \\
\hline Nuclei & $0, \mathrm{~g} / \mathrm{cm}^{2}$ & $2, \mathrm{~g} / \mathrm{cm}^{2}$ & $5, \mathrm{~g} / \mathrm{cm}^{2}$ & $20, \mathrm{~g} / \mathrm{cm}^{2}$ \\
\hline${ }^{6} \mathrm{He}$ & 0.0 & $9.1 \times 10^{1}$ & $2.1 \times 10^{2}$ & $5.6 \times 10^{2}$ \\
\hline${ }^{8} \mathrm{Li}$ & 0.0 & $1.0 \times 10^{3}$ & $2.2 \times 10^{3}$ & $5.4 \times 10^{3}$ \\
\hline${ }^{9} \mathrm{Li}$ & 0.0 & $3.0 \times 10^{1}$ & $7.2 \times 10^{1}$ & $1.8 \times 10^{2}$ \\
\hline${ }^{10} \mathrm{Be}$ & $8.8 \times 10^{3}$ & $9.1 \times 10^{3}$ & $9.3 \times 10^{3}$ & $8.8 \times 10^{3}$ \\
\hline${ }^{14} \mathrm{C}$ & 0.0 & $7.1 \times 10^{2}$ & $1.7 \times 10^{3}$ & $3.4 \times 10^{3}$ \\
\hline${ }^{18} \mathrm{O}$ & $9.9 \times 10^{3}$ & $9.3 \times 10^{3}$ & $8.4 \times 10^{3}$ & $5.1 \times 10^{3}$ \\
\hline${ }^{26} \mathrm{Al}$ & $5.7 \times 10^{2}$ & $7.6 \times 10^{2}$ & $9.4 \times 10^{2}$ & $1.0 \times 10^{3}$ \\
\hline${ }^{36} \mathrm{Cl}$ & $8.1 \times 10^{2}$ & $7.7 \times 10^{2}$ & $7.1 \times 10^{3}$ & $4.4 \times 10^{2}$ \\
\hline
\end{tabular}




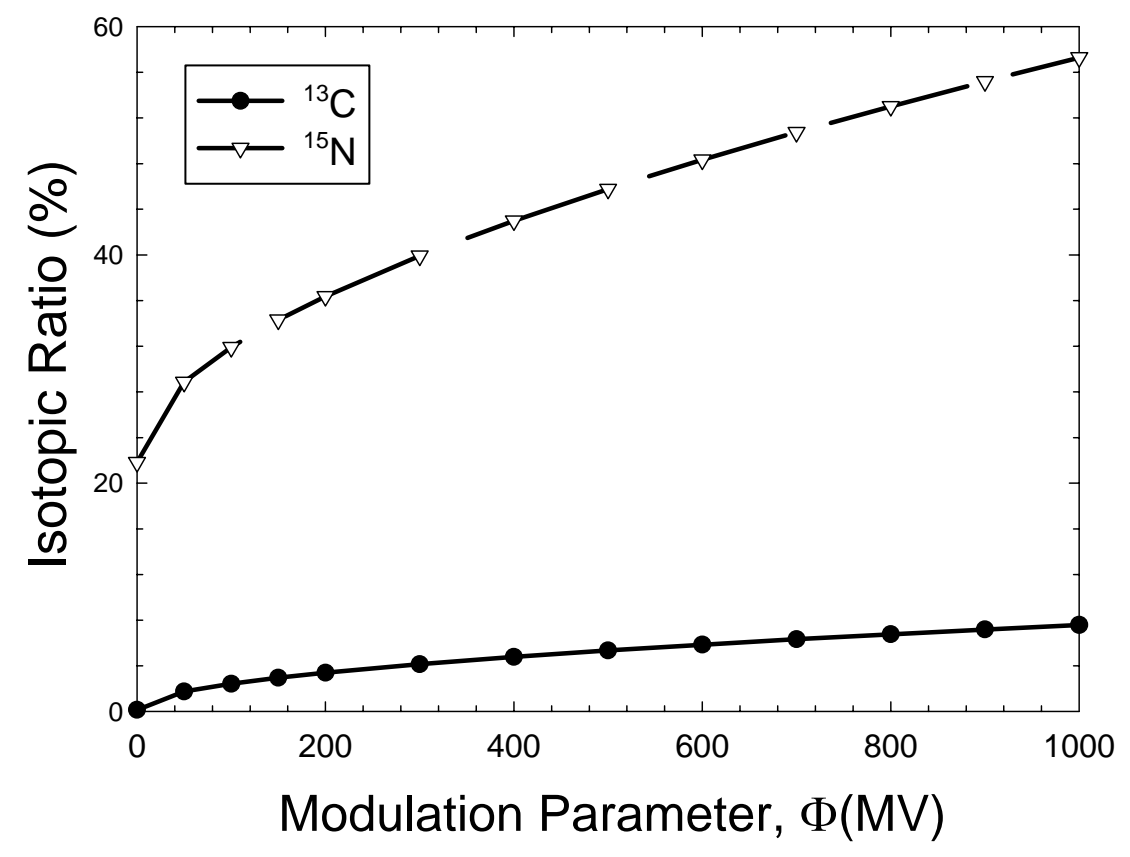

Figure 1: Parametric model for describing the change in isotopic composition with the solar modulation parameter, $\Phi(\mathrm{MV})$. 


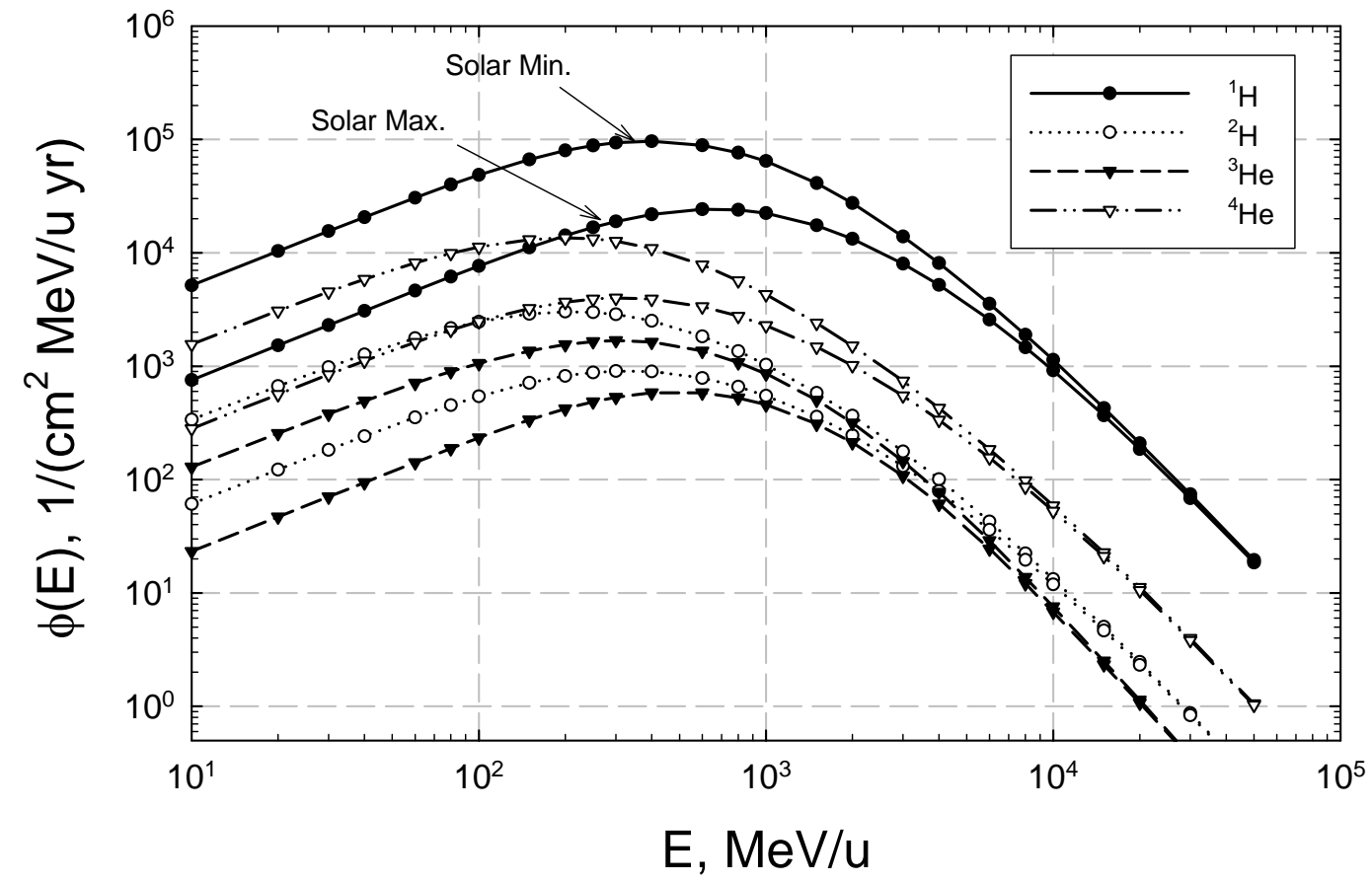

Figure 2a: Energy spectra for hydrogen and helium isotopes near solar minimum ( $\Phi=428 \mathrm{MV})$ and solar maximum ( $\Phi=1050 \mathrm{MV})$.

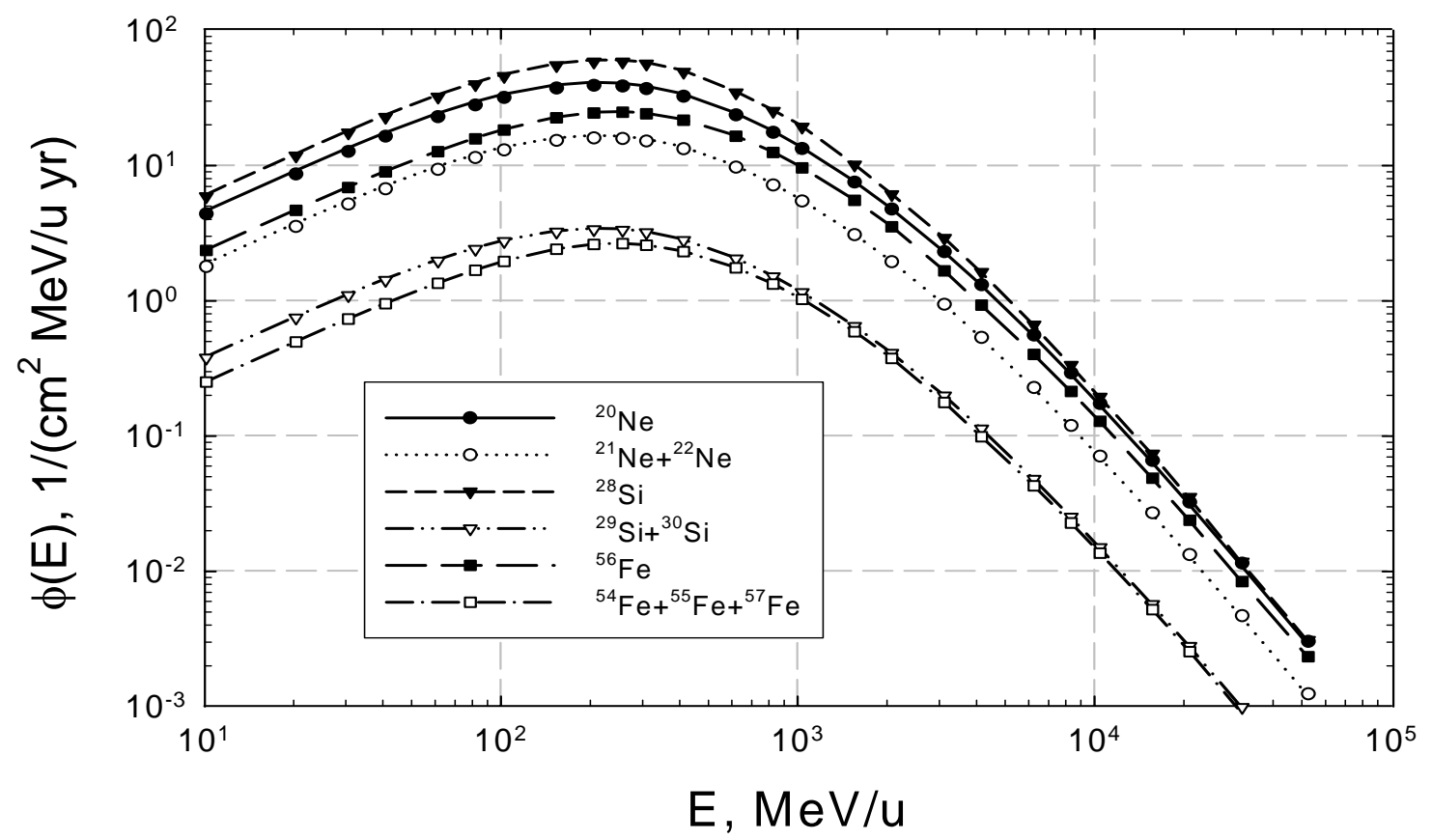

Figure 2b: Energy spectra for $\mathrm{Ne}, \mathrm{Si}$, and Fe isotopes near solar minimum ( $\Phi=428)$ showing contributions from different isotopes to primary GCR composition. 


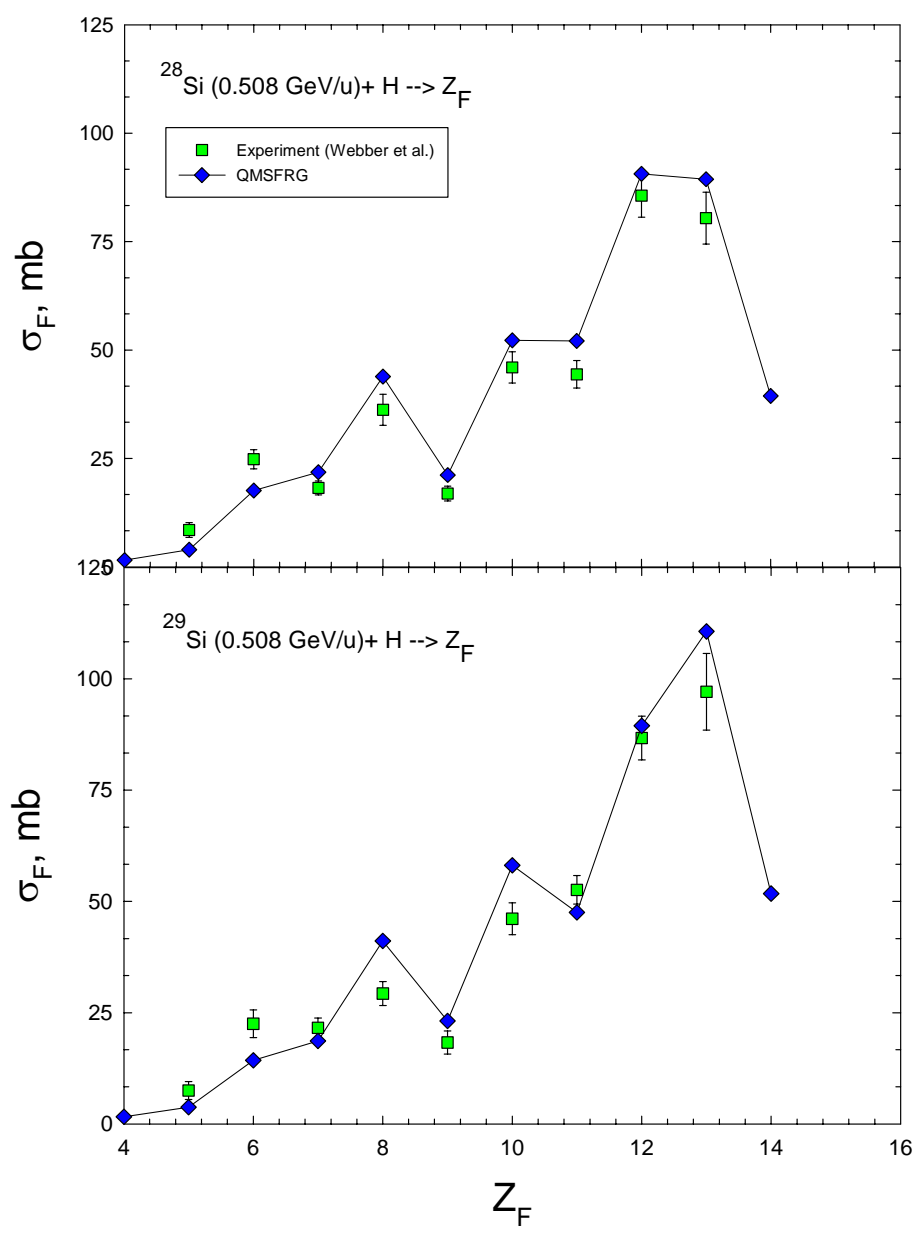

Figure 3: Comparisons of the QMSFRG model to experiment for elemental fragment distribution for ${ }^{28} \mathrm{Si}$ and ${ }^{29} \mathrm{Si}$ on $\mathrm{H}$ at $0.51 \mathrm{GeV} / \mathrm{u}$. Experimental data from Webber et al. (1998). 


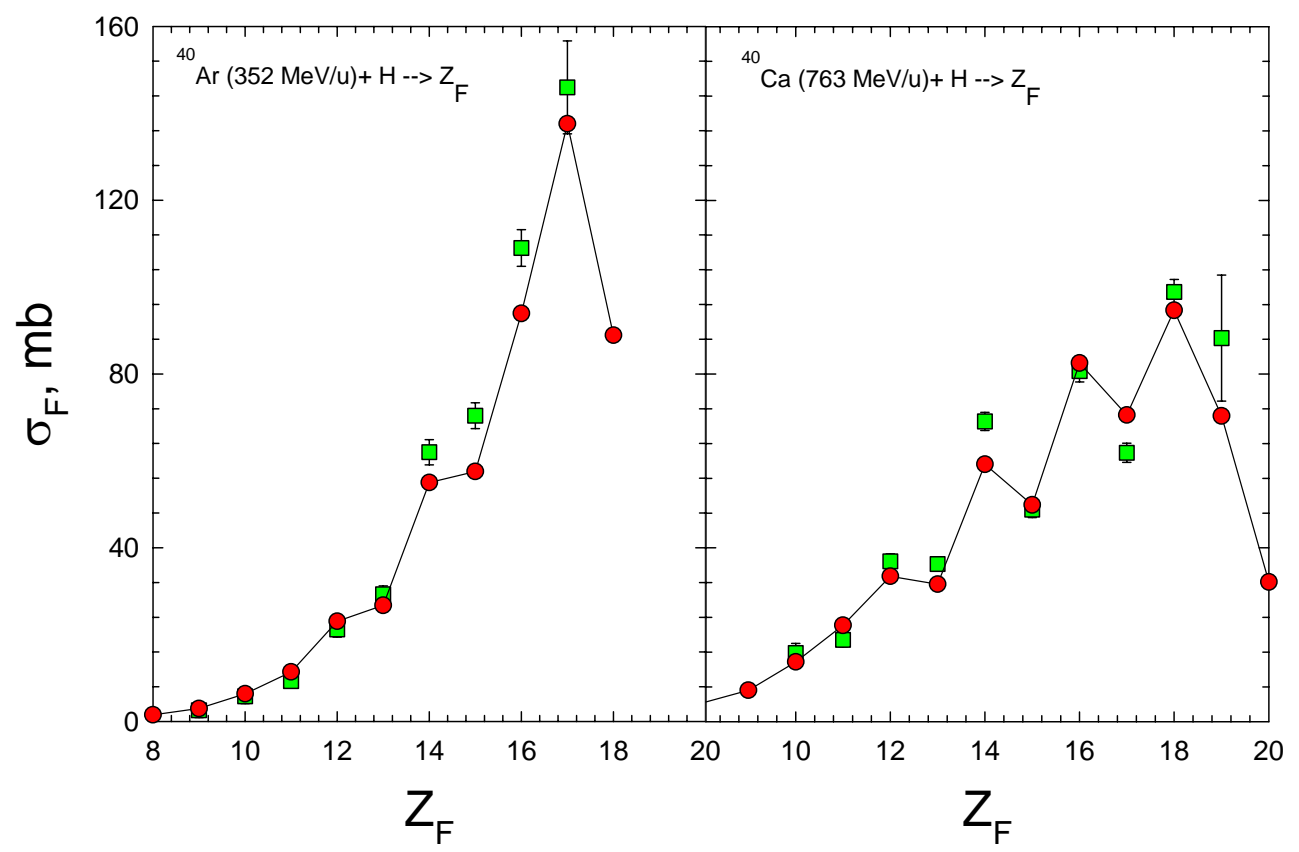

Figure 4a: Comparisons of the QMSFRG model to experiment for fragmentation of (left) ${ }^{40} \mathrm{Ar}$ on $\mathrm{H}$ at $0.35 \mathrm{GeV} / \mathrm{u}$ and for (right) ${ }^{40} \mathrm{Ca}$ on $\mathrm{H}$ at $0.76 \mathrm{GeV} / \mathrm{u}$ (Knott et al. (1996)).

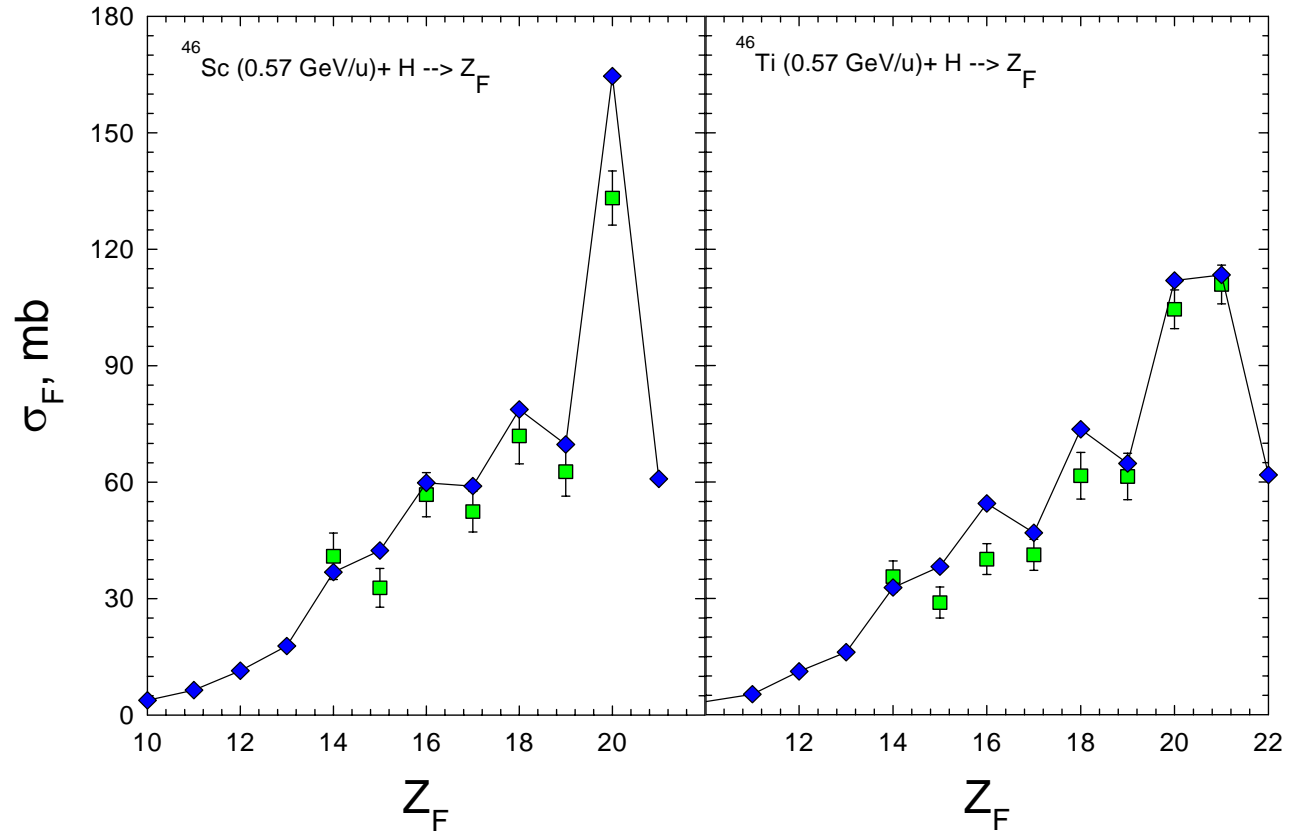

Figure 4b: Comparisons of the QMSFRG model to experiment for fragmentation of (left) ${ }^{46} \mathrm{Sc}$ on $\mathrm{H}$ at $0.57 \mathrm{GeV} / \mathrm{u}$ and for (right) ${ }^{46} \mathrm{Ti}$ on $\mathrm{H}$ at $0.57 \mathrm{GeV} / \mathrm{u}$ (Webber et al. (1998)). 

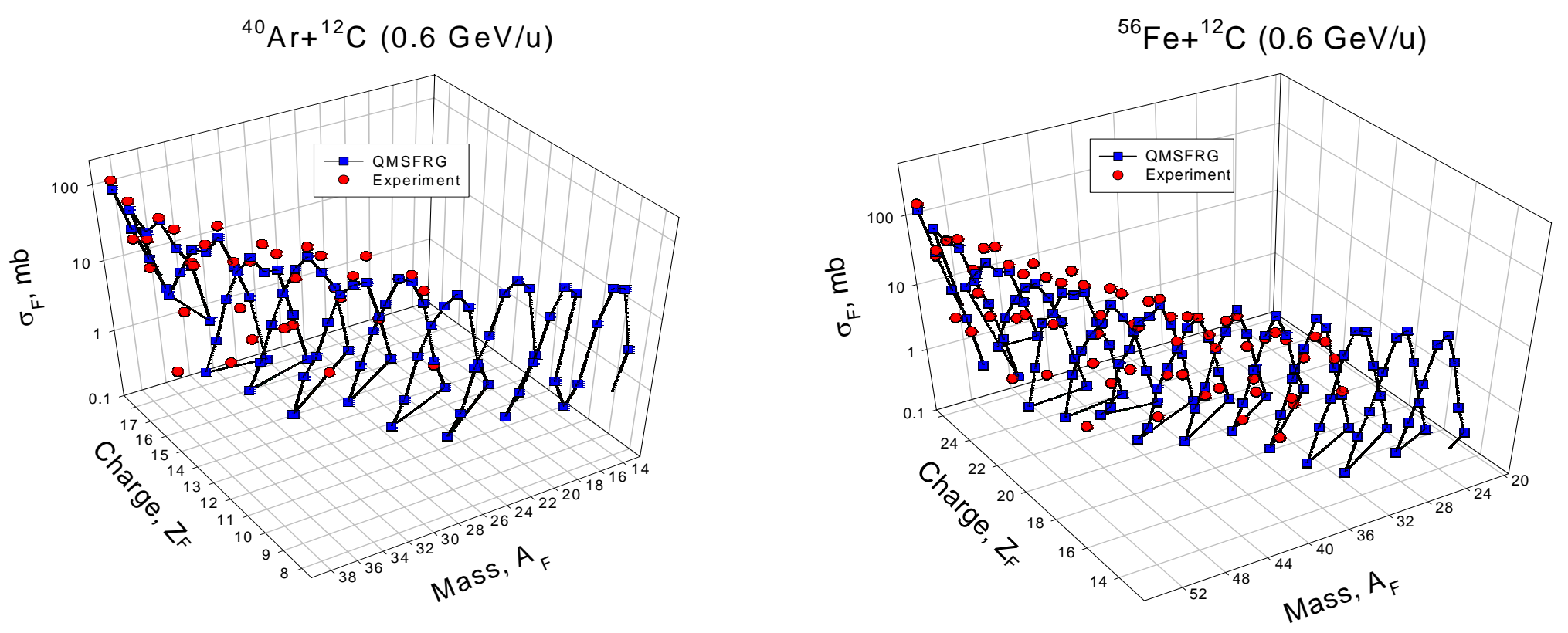

Figure 5: Comparisons of the QMSFRG model to experiment for isotopic distribution of fragments for a) ${ }^{40} \mathrm{Ar}$ on ${ }^{12} \mathrm{C}$ at $0.6 \mathrm{GeV} / \mathrm{u}$. and b) ${ }^{56} \mathrm{Fe}$ on ${ }^{12} \mathrm{C}$ interactions at $0.6 \mathrm{GeV} / \mathrm{u}$. Experimental data from Webber et al. (1990b). 

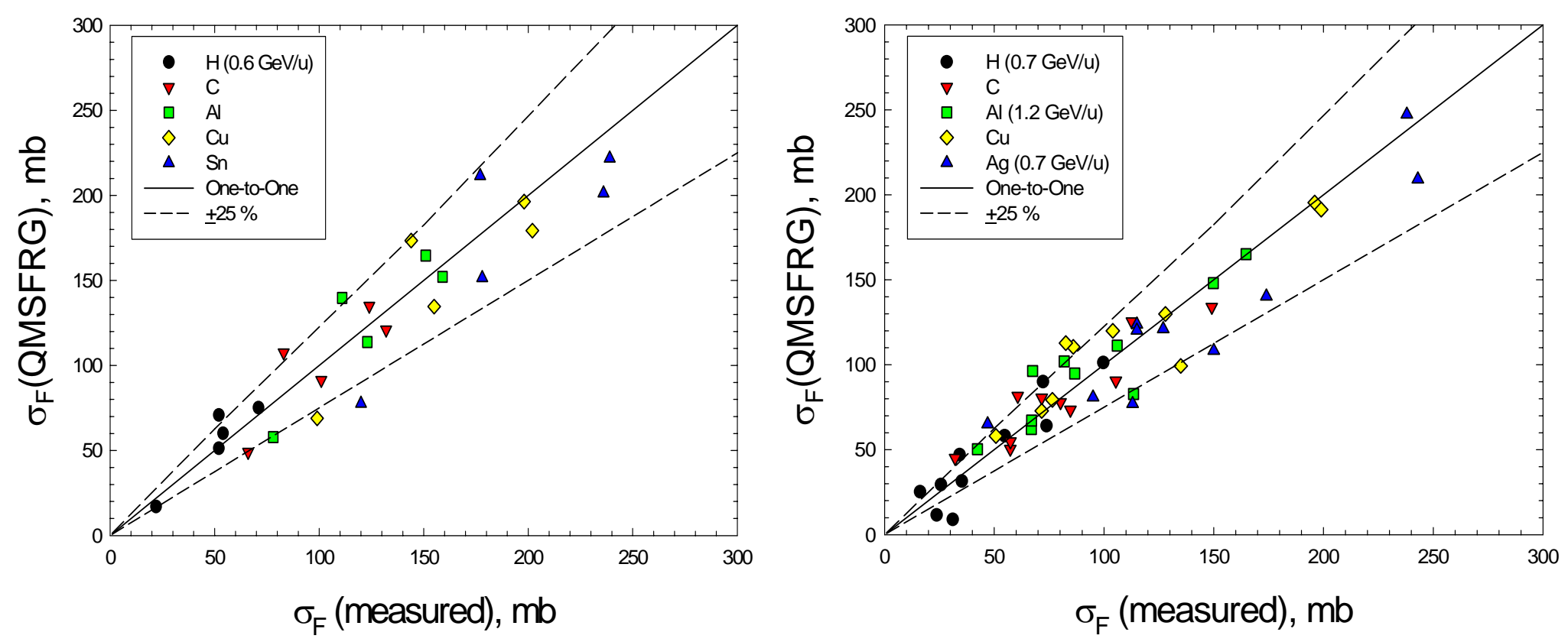

Figure 6: a) Comparison of QMSFRG to data of Zeitlin et al. (2001) for ${ }^{20} \mathrm{Ne}$ fragmentation on several targets at $0.6 \mathrm{GeV} / \mathrm{u}$. And b) comparison to data of Brechtmann et al. (1997) for ${ }^{32} \mathrm{~S}$ fragmentation on several targets at 0.7 or $1.2 \mathrm{GeV} / \mathrm{u}$. 


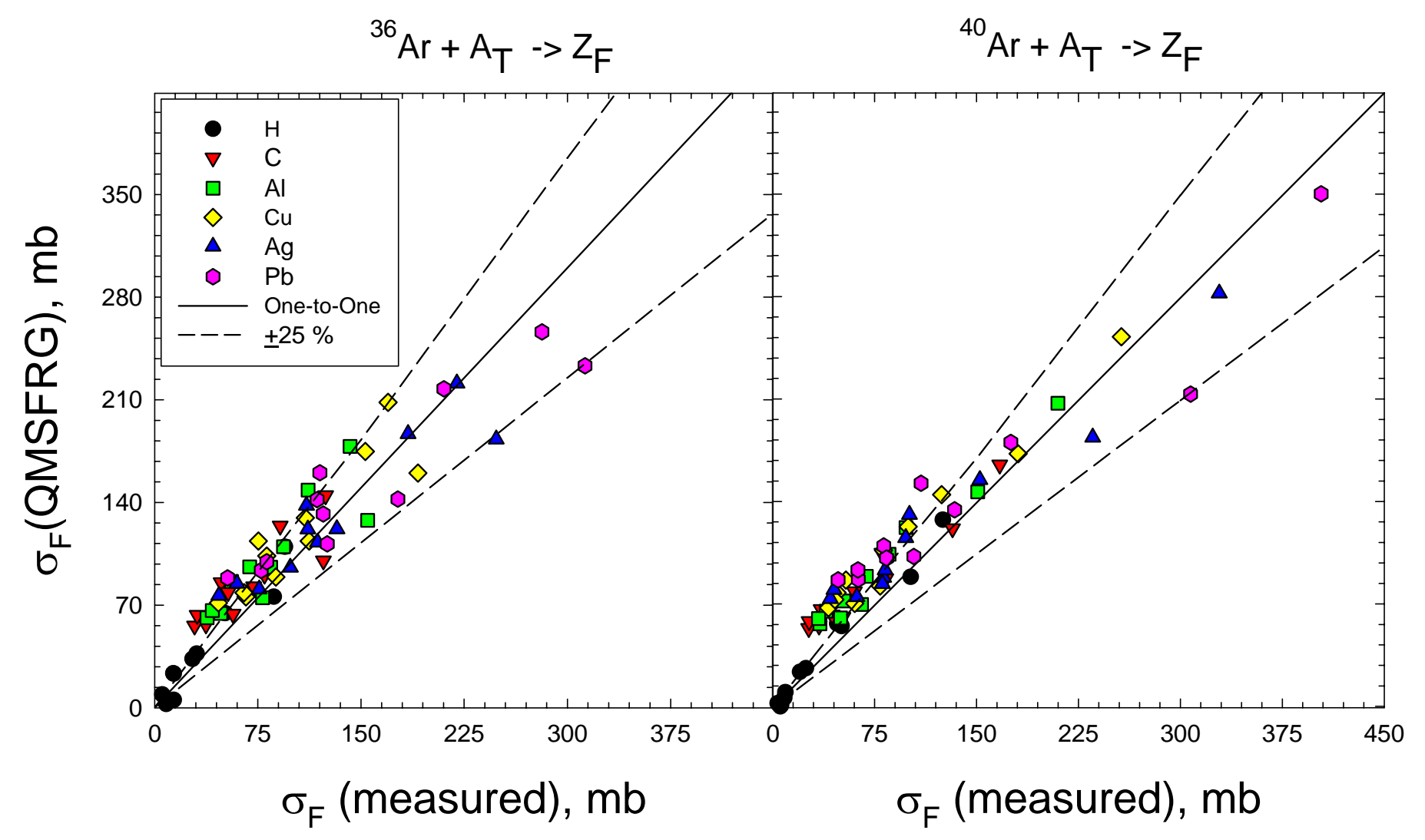

Figure 7: Comparison of QMSFRG to data of lancu et al. (2005) for ${ }^{36} \mathrm{Ar}$ or ${ }^{40} \mathrm{Ar}$ fragmentation on several targets at $0.34 \mathrm{GeV} / \mathrm{u}$. 


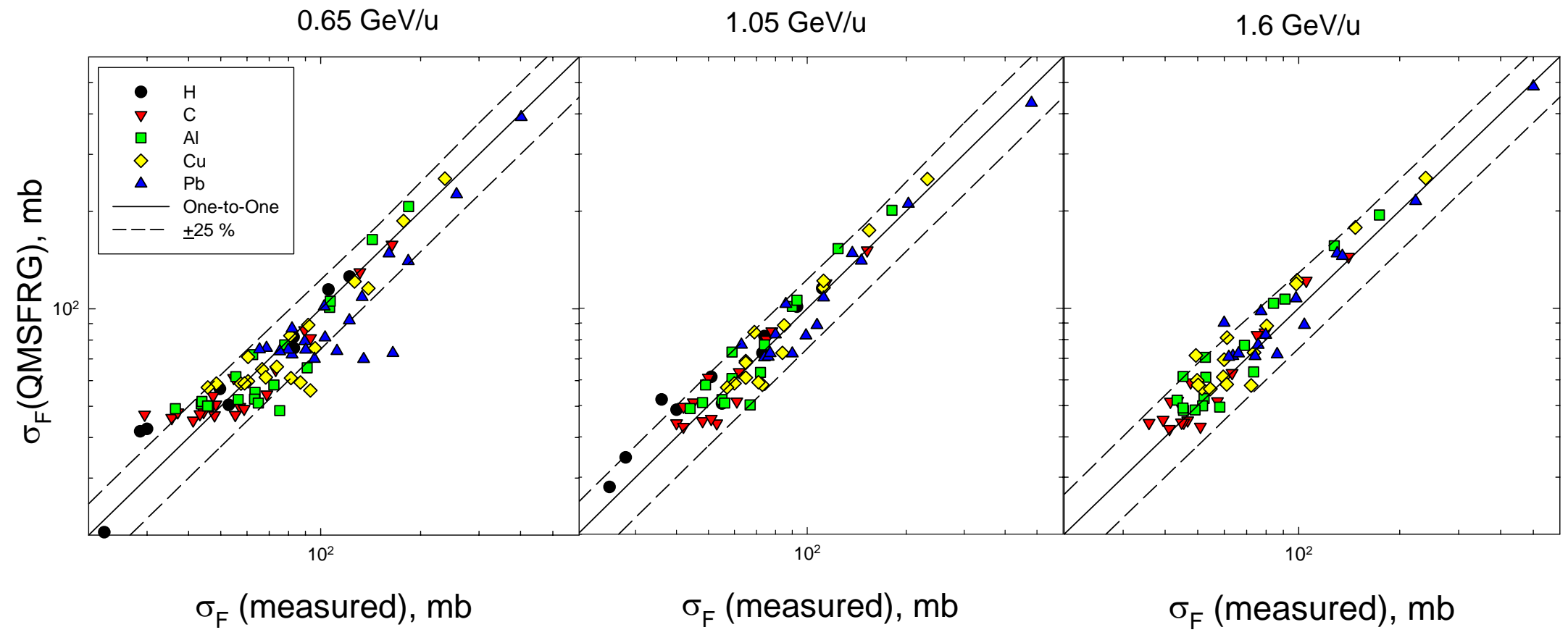

Figure 8: Comparison of QMSFRG model to experimental data for ${ }^{56} \mathrm{Fe}$ fragmentation on several targets at $0.65 \mathrm{GeV} / \mathrm{u}$ (Flesch et al. (1999)), $1.05 \mathrm{GeV} / \mathrm{u}$ (Zeitlin et al. (1997)), and 1.6 GeV/u (Cummings et al., 1990). 


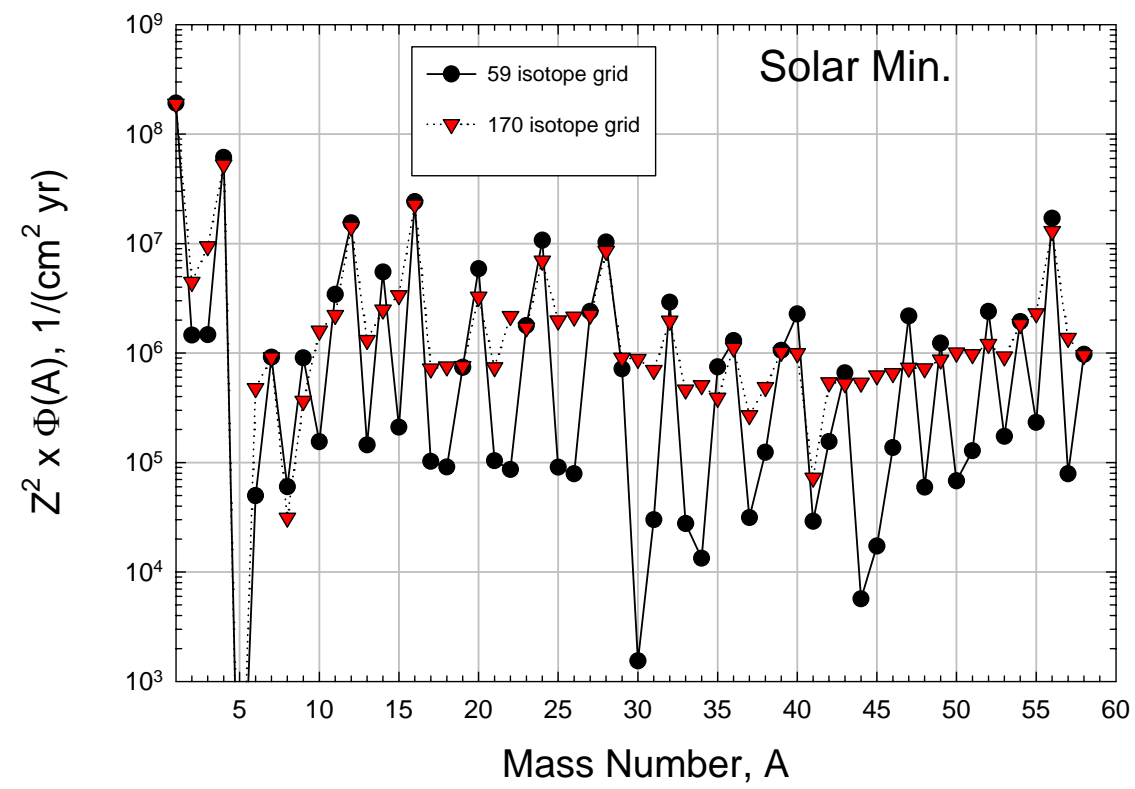

Figure 9: Comparisons of results from the HZETRN Code for the mass flux distribution behind $5 \mathrm{~g} / \mathrm{cm}^{2}$ of aluminum shielding for solar minimum conditions comparing transport with the reduced 59-isotope grid to transport with a full 170-isotope grid (proton fluence shown for $A=1)$. 


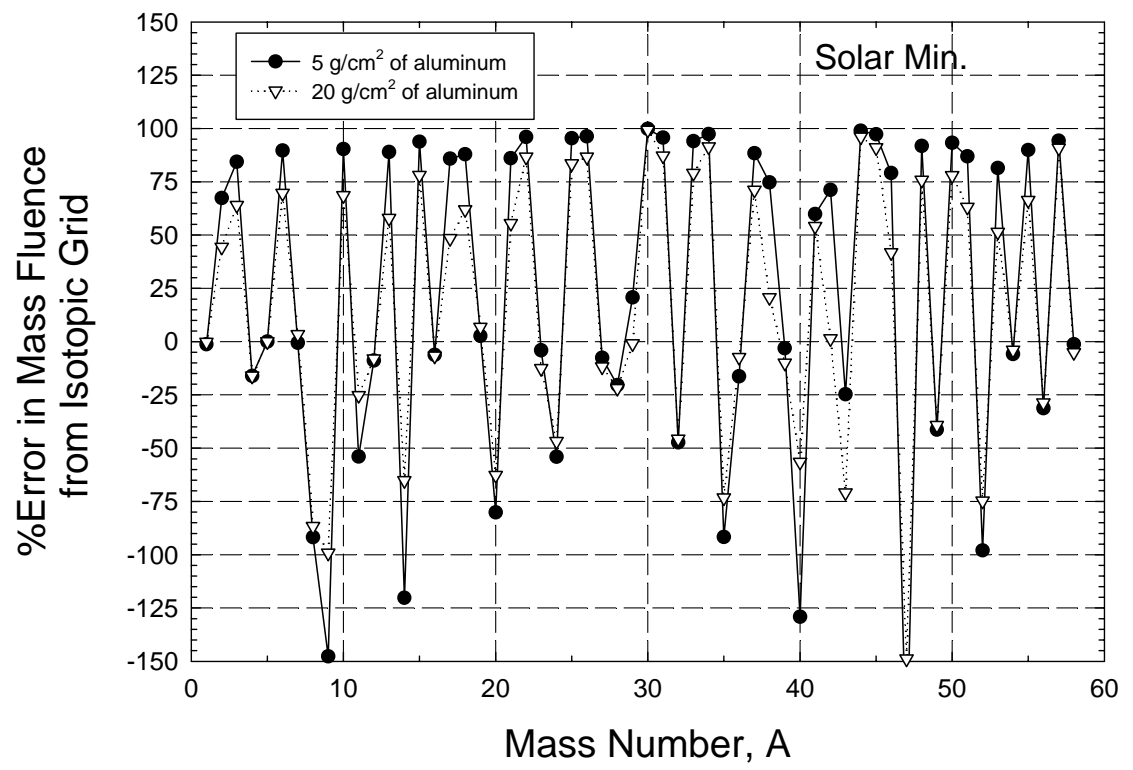

Figure 10a: Comparisons of the error that results from the HZETRN Code for the mass fluence distribution near solar minimum when using a reduced 59-isotope grid compared to transport with a 170-isotope grid.



Figure 10b: Comparisons of the error that results from the HZETRN Code for the elemental fluence distribution near solar minimum when using a reduced 59-isotope grid compared to transport with a 170-isotope grid. 


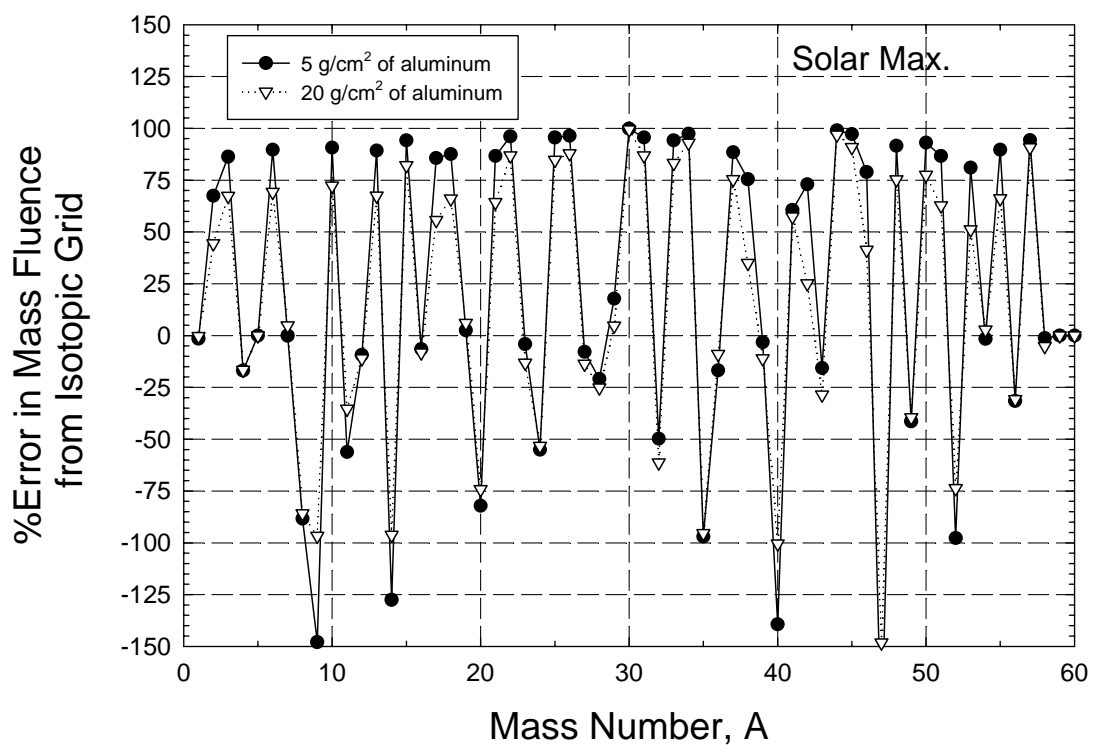

Figure 11a: Comparisons of the error that results from the HZETRN code for the mass fluence distribution near solar maximum when using a reduced 59-isotope grid compared to transport with a170-isotope grid.



Figure 11b: Comparisons of the error that results from the HZETRN code for the elemental fluence distribution near solar maximum when using a reduced 59-isotope grid compared to transport with a170-isotope grid. 